\title{
MAPPING INDIAN DISTRICTS ACROSS CENSUS YEARS, 1971-2001
}

Hemanshu Kumar

Email: hemanshu@iesdelhi.org IES Abroad, Delhi

Rohini Somanathan

Email: rohini@econdse.org Delhi School of Economics

University of Delhi

Working Paper No. 176 


\title{
Mapping Indian Districts Across Census Years, 1971-2001
}

\author{
Hemanshu Kumar and Rohini Somanathan
}

March 2009

\section{Introduction}

The Indian states have been the standard unit of analysis for research on India that uses official data sources. For many empirical questions, states are a natural starting point because state governments set political agendas and budgets and administer a wide range of services. In addition, the boundaries of many states have been unchanged for over half a century and those of all major states were largely unchanged between 1971 and 2000. This stability has resulted in the relatively easy construction and use of panel data sets at the state level and these data have been used to ask a variety of questions relating to the effectiveness of public policy.

The use of more disaggregated district data allows the study of outcomes across regions with similar historical contexts and political regimes. States have an average of 20 districts, so district level panels can also be much larger. Most district-level studies however have relied on cross-sectional analysis because district comparisons over time are complicated by multiple boundary changes. Between 1971 and 2001, the number of districts increased from 356 to 593, a rise of about $67 \%$. The purpose of this paper is to provide information on boundary changes across districts that will facilitate the construction of district-level panel data sets.

We use population data from the state and central volumes of the Census of India to document changes in district boundaries between 1971 and 2001. For each decade during the 1971-2001 period, we classify districts into three categories: those with unchanged boundaries, those created by partitioning existing districts and finally, districts whose current boundaries were located in multiple districts at the time of the previous census. We find that 136 of the 356 Indian districts in 1971 (38\%) were unaffected by boundary changes over the subsequent three decades, 79 districts $(22 \%)$ were cleanly partitioned into multiple districts over the same period, and the 
remaining 141 districts experienced more complex changes. Unchanged districts obviously pose no problem for the construction of panel data and the number of these districts can be quite large for short panels. For partitioned districts we provide population weights that permit the construction of panels using boundaries of either later or earlier census years as the base.

For districts that are neither unchanged nor partitioned it is in general only possible to generate accurate population weights across adjacent census years. We provide these weights separately for the three periods: 1971-1981, 1981-1991, and 1991-2001. In addition, we amalgamate neighbouring districts into composite regions with unchanged boundaries between each census year and 2001. These composite regions, along with the unchanged and partitioned districts, give us the complete set of geographical units with unchanged boundaries between any census year and 2001.

The following section provides details on data sources and our methods and compares these to those used by other studies relying on multi-year district data. Section 3 summarises some basic patterns. Section 4 concludes with some caveats on using our data and points to the type of work needed to construct district-level series over long time periods.

\section{Data Sources and Methods}

Our mapping of districts across 1971-2001 is based on tables provided as part of a publication on General Population Tables (Part II-A). These tables are published both in the state volumes as well as the national volumes. The national volumes document territorial changes at the district level, while individual state volumes also provide details of intra-district changes.

We use three primary sources from national volumes of the General Population Tables for all census years from 1971 to 2001. First, Appendix-1 to Table A-1 provides a detailed statement of territorial units at the time of the current census and the changes in territorial boundaries during the preceding decade. For each affected territorial unit, it lists the regions added and subtracted, along with their areas in square kilometres.

Our second source is the Appendix to Table A-2 in the same publication. This appendix lists the area and population of all districts as per their current boundaries. For each district it also provides the population in the previous census adjusted to the current boundaries. This would 
be the number obtained by netting out the population of the areas transferred in and out of the district. In a large number of cases, when a district is partitioned or only affected by one area transfer, this table is sufficient to deduce the population of individual regions transferred.

Our third source is the footnotes to the Appendix to Table A-2. These footnotes detail individual transfers between affected districts, together with the number and year of the government notification that effected the transfer. A typical entry from a footnote on p. 142 of the Census 2001 national volume (Government of India, 2005) reads as follows:

58 villages with an area of $147.29 \mathrm{sq} . \mathrm{km}$. and population of 57,365 were transferred to Khamanon tahsil of newly formed Fatehgarh Sahib district vide Punjab Govt Notification No. 2/3/92-RE.II(i)/4412 dated 9.4.92.

Occasionally, the above sources from the national volumes are insufficient to figure out individual boundary changes. ${ }^{1}$ In these cases, we took recourse to the more detailed tables published in the state volumes. ${ }^{2}$ There are also some small areas close to state borders whose jurisdiction is disputed. There is no systematic approach to classifying these areas. They are sometimes included in the population figures for both states and sometimes excluded completely. We simply use the population figures reported by the census volumes without attempting any adjustments. ${ }^{3}$

We refer to a district $B$ as a child of another district $A$, if any inhabited areas have been transferred from $A$ to $B$ during the period under consideration. District $A$ is referred to as a parent of $B$. Unchanged districts are single parent and single child districts and partitioned districts share a single parent. For these two classes of districts it is straightforward to construct a district panel based on district boundaries corresponding to 1971 because populations for boundaries corresponding to the parent district are simply the sum of child populations. To illustrate, the district of Muzaffarpur in Bihar in 1971 is partitioned into the three districts Muzaffarpur, Sitamarhi and Vaishali in 1972 and Sitamarhi is further partitioned in 1994 to form Sheohar district. To obtain a time series on (say) the literate population of Muzaffarpur as per its 1971 boundaries, one would simply aggregate the literate population in the child districts for subsequent census years. It is also possible to create a panel based on district boundaries of later census years if we make certain assumptions about the distribution of the population. In the above example, an estimate of the number of literates in Sheohar in 1971 can be obtained by multiplying the data for Muzaffarpur in that year, by the product of two terms. The first is the fraction of the 1971 Muzaffarpur population that was in Sitamarhi by 1981, and the second is the fraction of the Sitamarhi population in 1991 which fell within the boundaries of 
Sheohar. The greater the uniformity in the distribution of the literate population across the original district, the more accurate would be a time series generated in this manner.

When a district has more than one parent, we cannot use the above procedure to obtain district panels spanning multiple census years. For example, a large part of present-day Champawat district was formed from Champawat tahsil of Pithoragarh district in 1997 (with the remaining part coming from Nainital district). Champawat tahsil was part of Almora district until Census 1971, and was transferred to Pithoragarh district only during the 1971-1981 period. No part of the area within Pithoragarh's boundaries in 1971 was part of Champawat in 2001. Yet, if we mechanically and successively applied weights based on population shares over the census years, we would attribute $59 \%$ of the population of present-day Champawat as coming from Pithoragarh in $1971 .^{4}$

In the above example, an entire tahsil was transferred across districts. However a large number of boundary changes involve a portion of a tahsil or taluk being transferred to a different district. In such cases, the tables tell us the number of villages that were transferred, but do not identify the villages themselves. This makes it impossible to track the transferred region across multiple census years. For these reasons, we only provide weights across adjacent census years for districts with multiple parents. To create longer panels, albeit with some error, our tables must be combined with a visual examination of the maps described below.

Another approach to creating long panels which include all parts of the country is to amalgamate districts into composite regions which experience no inter-regional transfers. To generate these, each parent district is put in the same region as all its child districts. To illustrate with Andhra Pradesh, the state had 21 districts in 1971 and the boundaries of 13 of these were intact in 2001. The remaining 8 districts can be grouped into 5 composite regions with unchanged regional boundaries over the thirty year period (Table 8). As a result, there are a total of 18 regions of Andhra Pradesh which can form the basis for a 1971-2001 panel data set for the state.

In contrast to our methods, most existing studies that have used multi-year district level data have combined a visual comparison of district maps with some information on areas transferred. Murthi et al. (2001) use administrative maps to create a correspondence between 1981 and 1991 districts. Banerjee and Iyer (2005) collapse modern districts into 1961 districts to examine the effect of historical land tenure systems on agricultural productivity in subsequent years. Banerjee and Somanathan (2007) compare 1931 districts with those in the 1961-1991 period and also map current parliamentary constituencies to districts in the latter period to explore the relationship between constituency demographics and the availability of public goods. Most 
recently, Bharadwaj et al. (2008) map districts of British India in 1931 with corresponding areas in Pakistan and India in 1951 to study labour migration induced by the Partition of India.

Maps for successive census years are available in the Indian Administrative Atlas 1872-2001 (Government of India, 2004) published as part of the documentation of Census 2001. Map No. 15 in this publication provides a list of newly created districts in each decade between 1951 and 2001. Appendix I tracks changes in the spellings of district names in each decade, while Appendix III provides an "Equivalence Table of Administrative Divisions 1961-2001". 5

While these administrative maps are extremely useful in pointing out the principal antecedents of districts over long periods of time, visual comparisons of district boundaries based on these maps is necessarily coarse. Differences in population density are ignored and districts which either lose or receive small areas may appear unchanged. To illustrate, Murthi et al. (2001) use administrative maps to classify 368 districts as being unchanged between 1981 and 1991, and another 42 districts as partitioned into two or three 1991 districts each (see their Table 2). Our own analysis reveals that there were only 313 districts that were unchanged between 1981 and 1991, and another 23 that were partitioned. Many of the differences in classification arise because small transfers are neglected when maps are used. If for example, we ignore transfers of up to $5 \%$ of the original district's population, we get 358 unchanged districts and 35 districts being partitioned between 1981 and 1991. These errors of mis-classification are likely to get compounded in studies that cover longer time periods.

We are aware of one other study that relies on the population table we have used in this paper. Reddy (1988) in his historical study of changes in agricultural labour uses them to isolate 98 regions with unchanged boundaries over the 1881-1981 period. Our contribution lies in using these figures to construct a comprehensive classification of district-level boundary changes for the 1971-2001 period.

\section{$3 \quad$ Summary of Findings}

Table 1 lists the number of districts in each state for each census year. The total number of Indian districts went up by $15.7 \%$ and $13 \%$ respectively in the first two decades and then by 27.3\% during the 1991-2001 decade. New districts were created at very uneven rates across the Indian states. No new districts were created in Gujarat and Orissa until 1991 and only one or two 
new districts appeared in several other states (Himachal Pradesh, Punjab, Rajasthan, Madhya Pradesh, West Bengal, Andhra Pradesh and Karnataka). Most boundary changes during the 1971-1991 period were concentrated in Tamil Nadu, Bihar, the north-eastern states and the union territories. In the subsequent decade, most states contributed to the substantial increase in the number of districts. The only exceptions were the states of Assam, Himachal Pradesh, Andhra Pradesh and Kerala, where the number of districts remained unchanged between 1991 and 2001.

Tables 2 and 3 show how districts can be placed in our three-fold classification - those with unchanged boundaries, clean partitions of existing districts and a residual category of all remaining districts. Some states such as Bihar are good candidates for inclusion in multi-year panels because, even though the number of districts increased significantly, new districts were created by partitioning existing ones. In contrast, Haryana and Gujarat experienced a complex reorganisation of district boundaries. This makes it much harder to create long demographic panels for these states.

Table 4 lists the names of all districts whose boundaries remained unchanged throughout the 1971-2001 period. Table 5 lists all partitioned districts, and for each new district it provides the population share it received of the parent district. Table 6 contains districts that are neither unchanged nor partitioned. As argued in Section 2, for these districts it is only possible to define meaningful population weights for adjacent census years. Tables $6 a-6 \mathrm{c}$ provide these weights.

As discussed in Section 2, it is possible to create a 1971-2001 district panel for the entire country as long as we are willing to amalgamate the districts in Tables 6a-6c into composite regions with unchanged boundaries. Table 7 tells us the size of the panels that can be generated in this manner. For the whole country, we can define 267 such regions for the 1971-2001 period, 334 for 1981-2001 and 414 for 1991-2001.

Table 8 lists the composite regions individually. In some cases the complexity of underlying transfers means that such regions can be quite large. To cite the two largest composite regions by population for the period 1971-2001: the entire state of Punjab except Kapurthala district forms one composite region, while the entire state of Haryana forms part of one composite region that also includes two neighbouring districts (Bulandshahr and Meerut) of the state of Uttar Pradesh. In general, there are very few such regions that cross state boundaries. 


\section{Conclusion}

The purpose of this paper is to provide data on the composition of all Indian districts over the 1971-2001 period that can enable the construction of district panels. We conclude with a few caveats on using our data.

First, we have restricted ourselves to an examination of population shares and have neglected changes in district areas. The resulting classification is a natural one for the study of demographic questions but may not be the most appropriate for studies of land-use or climate, where weights based on areas transferred may seem more appropriate. Our main reason for not supplementing population changes with a study of areas transferred is the difficulty in obtaining a consistent time series of district areas. Different administrative authorities are responsible for the compilation of district and sub-district areas; the Office of the Surveyor General provides district areas while areas of jurisdictional boundaries below the district level (blocks, tahsils, villages) are based on numbers provided by land revenue authorities and municipal bodies (see Government of India, 2005, p. 25). These two sets of figures are often inconsistent.

Second, we have limited ourselves to changes in boundaries across census years. Much of the research on India uses household survey data from the National Sample Survey, the National Family Health Survey and other sources. The years for which these data are collected do not match census years. To create a panel based on these data sets, researchers can use the footnotes to the Appendix to Table A-2 which we have mentioned in Section 2 because these notes contain the year in which each transfer was made. ${ }^{6}$

A final caveat relates to the creation of multi-year district panels. The information provided here only allows for these to be created for unchanged districts, single parent districts, and the amalgamated regions described Section 2 and listed in Table 8. For districts with multiple parents, it is not in general possible to use data on transfers in successive years to generate population weights over longer periods, as discussed in Section 2. If the data presented here are used for this purpose, they should certainly be combined with an examination of maps from the Census publications to minimise errors. Ultimately the only way to create error-free panels for all districts is to track the movement of villages across district boundaries. This is possible but time-consuming for the years before 1991 when census village data was not available in digital form. We leave this for future work. 


\section{Notes}

${ }^{1}$ This is the case for changes in Himachal Pradesh during 1971-81, Haryana during 1981-1991, Assam during 1971-91, and Manipur during 1981-91.

${ }^{2}$ In one case, Haryana, both the national and state volumes are inadequate in identifying boundary changes during 1981-1991. Several transfers during this period in Haryana involved small numbers of villages and neither central nor state volumes list the population of transferred villages. As noted at the bottom of Table $6 \mathrm{~b}$, our figures for selected districts in Haryana over this period are, as a result, approximate.

${ }^{3}$ For example, due to the changing course of the river Ganga, the status of 18 villages has been under dispute between the states of West Bengal and Jharkhand. The populations of these villages for 2001 have been included in both Maldah district of West Bengal and Sahibganj district of Jharkhand. (Government of India, 2005, p. 24) In contrast, an area of 13 sq. km. which has been long disputed by Pondicherry and Andhra Pradesh has been excluded from the population of both regions. (Government of India, 2005, p. 169)

${ }^{4}$ From Tables $6 \mathrm{a}-6 \mathrm{c}$, the product of the population weights is computed as $75.57 \% \times 100 \% \times 78.44 \%=59.28 \%$

${ }^{5}$ More detailed maps are also available from three other sources: (i) the state-wise Administrative Atlas series published for the 2001 Census; (ii) the state-wise Census Atlas series published with every Census since 1961; and (iii) the District Census Handbooks, of which there is one volume per district in every census year.

${ }^{6}$ However, these notes are sometimes incomplete. Aside from the previously mentioned case of Haryana during 1981-1991, information on the year of the transfer is missing in the tables for Uttar Pradesh and Arunachal Pradesh during 1971-1981 and for West Bengal during 1981-1991.

\section{References}

Banerjee, Abhijit, and Lakshmi Iyer (2005) 'History, Institutions, and Economic Performance: The Legacy of Colonial Land Tenure Systems in India.' The American Economic Review 95(4), 1190-1213

Banerjee, Abhijit, and Rohini Somanathan (2007) 'The Political Economy of Public Goods: Some Evidence from India.' Journal of Development Economics 82(2), 287-314 
Bharadwaj, Prashant, Asim Khwaja, and Atif Mian (2008) 'The Big March: Migratory Flows after the Partition of India.' Economic and Political Weekly 43(35), 39-49

Government of India (1996) General Population Tables and Primary Census Abstract Census of India 1991: Series 8: Haryana (Chandigarh: Director of Census Operations, Haryana)

- (2004) India Administrative Atlas 1872-2001: A Historical Perspective Census of India 2001 (New Delhi: Office of the Registrar General \& Census Commissioner, India). Product No. 00-068-2001-Cen-Book.

- (2005) General Population Tables: India, States and Union Territories, Part-I Census of India 2001 (New Delhi: Office of the Registrar General \& Census Commissioner, India). Product No. 00-130-2001-Cen-Book.

Murthi, Mamta, P. V. Srinivasan, and S. V. Subramanian (2001) 'Linking Indian Census with National Sample Survey.' Economic and Political Weekly 36(9), 783-792

Reddy, Rammanohar (1988) 'The Incidence of Agricultural Labourers in India: Variations Over Time and Space, 1881-1981.' PhD dissertation, Jawaharlal Nehru University, Delhi 
Table 1. Number of Districts in Each Census Year

\begin{tabular}{|c|c|c|c|c|}
\hline \multirow[t]{2}{*}{ Census 2001 State/UT } & \multicolumn{4}{|c|}{ Number of Districts } \\
\hline & 1971 & 1981 & 1991 & 2001 \\
\hline [1] & {$[2]$} & {$[3]$} & {$[4]$} & {$[5]$} \\
\hline Andaman \& Nicobar Islands & 1 & 2 & 2 & 2 \\
\hline Andhra Pradesh & 21 & 23 & 23 & 23 \\
\hline Arunachal Pradesh & 5 & 9 & 11 & 13 \\
\hline Assam & 10 & 10 & 23 & 23 \\
\hline Bihar & 17 & 31 & 42 & 37 \\
\hline Chandigarh & 1 & 1 & 1 & 1 \\
\hline Chhattisgarh & & & & 16 \\
\hline Dadra \& Nagar Haveli & 1 & 1 & 1 & 1 \\
\hline Daman \& Diu & & & 2 & 2 \\
\hline Delhi & 1 & 1 & 1 & 9 \\
\hline Goa & 3 & 3 & 2 & 2 \\
\hline Gujarat & 19 & 19 & 19 & 25 \\
\hline Haryana & 7 & 12 & 16 & 19 \\
\hline Himachal Pradesh & 10 & 12 & 12 & 12 \\
\hline Jammu \& Kashmir & 10 & 14 & 14 & 14 \\
\hline Jharkhand & & & & 18 \\
\hline Karnataka & 19 & 19 & 20 & 27 \\
\hline Kerala & 10 & 12 & 14 & 14 \\
\hline Lakshadweep & 1 & 1 & 1 & 1 \\
\hline Madhya Pradesh & 43 & 45 & 45 & 45 \\
\hline Maharashtra & 26 & 26 & 30 & 35 \\
\hline Manipur & 5 & 6 & 8 & 9 \\
\hline Meghalaya & 2 & 5 & 5 & 7 \\
\hline Mizoram & & 3 & 3 & 8 \\
\hline Nagaland & 3 & 7 & 7 & 8 \\
\hline Orissa & 13 & 13 & 13 & 30 \\
\hline Pondicherry & 4 & 4 & 4 & 4 \\
\hline Punjab & 11 & 12 & 12 & 17 \\
\hline Rajasthan & 26 & 26 & 27 & 32 \\
\hline Sikkim ${ }^{\mathrm{a}}$ & 4 & 4 & 4 & 4 \\
\hline Tamil Nadu & 14 & 16 & 21 & 30 \\
\hline Tripura & 3 & 3 & 3 & 4 \\
\hline Uttar Pradesh & 54 & 56 & 63 & 70 \\
\hline Uttaranchal & & & & 13 \\
\hline West Bengal & 16 & 16 & 17 & 18 \\
\hline States $^{a}$ & 19 & 22 & 25 & 29 \\
\hline Union Territories & 10 & 9 & 7 & 6 \\
\hline Districts $^{\mathrm{a}}$ & 356 & 412 & 466 & 593 \\
\hline
\end{tabular}

${ }^{a}$ While Sikkim was not a part of the Union of India in 1971, later Indian Censuses allow us to trace boundary changes in Sikkim back till 1971. In this and all following tables, we use this information; however, the totals for states and districts in India do not include Sikkim in 1971.

\section{Notes:}

1. If a Union Territory is not further subdivided in any given census year, we count it as a single district in that year.

2. The Census did not cover Assam in 1981 and Jammu \& Kashmir in 1991 due to political unrest.

3. In November 2000, the states of Chhattisgarh, Jharkhand and Uttaranchal were created from Madhya Pradesh, Bihar and Uttar Pradesh, respectively. The UT of Goa, Daman \& Diu was separated into the state of Goa and the UT of Daman \& Diu in 1987. In the same year, the UTs of Mizoram and Arunachal Pradesh were also accorded statehood. Sikkim was added to the Union of India in 1975. Mizoram was created as a UT from Mizo district of Assam in 1972. In the same year, the UTs of Manipur and Tripura also acquired statehood.

4. Following Census documents, we count Delhi as a UT in 1971, 1981 and 1991, but as a state in 2001. The Autonomous State of Meghalaya was created on 2 April 1970, but it acquired full statehood only in 1972. Census 1971 documents list it as a state, and we follow this convention. 
Table 2. Summary of Intercensal Boundary Changes, 1971-1991

This table lists the number of districts that remained unchanged or were clean partitions of districts covered in the previous census year. The residual category is "Other".

\begin{tabular}{|c|c|c|c|c|c|c|}
\hline \multirow[t]{2}{*}{ State/UT } & \multicolumn{3}{|c|}{$1971-1981$} & \multicolumn{3}{|c|}{ 1981-1991 } \\
\hline & Unchanged & Partitions & Other & Unchanged & Partitions & Other \\
\hline [1] & {$[2]$} & [3] & [4] & {$[5]$} & {$[6]$} & {$[7]$} \\
\hline Andaman \& Nicobar Islands & 0 & 2 & 0 & 2 & 0 & 0 \\
\hline Andhra Pradesh & 16 & 2 & 5 & 18 & 0 & 5 \\
\hline Arunachal Pradesh & 1 & 4 & 4 & 5 & 4 & 2 \\
\hline Assam & 9 & 0 & 1 & 2 & 7 & 14 \\
\hline Bihar & 7 & 24 & 0 & 24 & 18 & 0 \\
\hline Chandigarh & 1 & 0 & 0 & 1 & 0 & 0 \\
\hline Dadra \& Nagar Haveli & 1 & 0 & 0 & 1 & 0 & 0 \\
\hline Daman \& Diu & & & & 2 & 0 & 0 \\
\hline NCT Delhi & 1 & 0 & 0 & 1 & 0 & 0 \\
\hline Goa & 3 & 0 & 0 & 0 & 2 & 0 \\
\hline Gujarat & 19 & 0 & 0 & 19 & 0 & 0 \\
\hline Haryana & 1 & 2 & 9 & 1 & 0 & 15 \\
\hline Himachal Pradesh & 5 & 3 & 4 & 10 & 0 & 2 \\
\hline Jammu \& Kashmir & 3 & 4 & 7 & 14 & 0 & 0 \\
\hline Karnataka & 19 & 0 & 0 & 16 & 2 & 2 \\
\hline Kerala & 4 & 0 & 8 & 6 & 2 & 6 \\
\hline Lakshadweep & 1 & 0 & 0 & 1 & 0 & 0 \\
\hline Madhya Pradesh & 40 & 2 & 3 & 45 & 0 & 0 \\
\hline Maharashtra & 26 & 0 & 0 & 16 & 0 & 14 \\
\hline Manipur & 2 & 0 & 4 & 3 & 0 & 5 \\
\hline Meghalaya & 0 & 5 & 0 & 5 & 0 & 0 \\
\hline Mizoram & 0 & 3 & 0 & 3 & 0 & 0 \\
\hline Nagaland & 0 & 7 & 0 & 1 & 0 & 6 \\
\hline Orissa & 13 & 0 & 0 & 13 & 0 & 0 \\
\hline Pondicherry & 4 & 0 & 0 & 4 & 0 & 0 \\
\hline Punjab & 4 & 0 & 8 & 7 & 0 & 5 \\
\hline Rajasthan & 18 & 0 & 8 & 21 & 2 & 4 \\
\hline Sikkim & 2 & 0 & 2 & 4 & 0 & 0 \\
\hline Tamil Nadu & 9 & 2 & 5 & 12 & 9 & 0 \\
\hline Tripura & 1 & 0 & 2 & 1 & 0 & 2 \\
\hline Uttar Pradesh & 49 & 2 & 5 & 41 & 8 & 14 \\
\hline West Bengal & 14 & 0 & 2 & 14 & 0 & 3 \\
\hline India & 271 & 62 & 79 & 313 & 54 & 99 \\
\hline
\end{tabular}

Notes:

1. In columns [2], [3] and [4], districts are listed as per 1981 jurisdictions, while in columns [5], [6] and [7], districts are listed as per 1991 jurisdictions.

2. The summary of intercensal boundary changes between 1991 and 2001 can be obtained from columns [8], [9] and [10] of Table 3. 
Table 3. Summary of Boundary Changes

This table lists the number of Census 2001 districts that had remained unchanged from, or were clean partitions of, districts covered in previous census years. The residual category is "Other".

\begin{tabular}{|c|c|c|c|c|c|c|c|c|c|c|}
\hline \multirow[t]{2}{*}{ Census 2001 State/UT } & \multicolumn{3}{|c|}{1971} & \multicolumn{3}{|c|}{1981} & \multicolumn{3}{|c|}{1991} & \multirow{2}{*}{$\begin{array}{c}\text { Total } \\
2001 \text { Districts }\end{array}$} \\
\hline & Unchanged & Partitions & Other & Unchanged & Partitions & Other & Unchanged & Partitions & Other & \\
\hline$[1]$ & {$[2]$} & {$[3]$} & [4] & {$[5]$} & {$[6]$} & [7] & {$[8]$} & [9] & [10] & {$[11]$} \\
\hline Andaman \& Nicobar Islands & 0 & 2 & 0 & 2 & 0 & 0 & 2 & 0 & 0 & 2 \\
\hline Andhra Pradesh & 13 & 0 & 10 & 18 & 0 & 5 & 21 & 0 & 2 & 23 \\
\hline Arunachal Pradesh & 0 & 7 & 6 & 4 & 6 & 3 & 9 & 4 & 0 & 13 \\
\hline Assam & 2 & 7 & 14 & 2 & 7 & 14 & 21 & 0 & 2 & 23 \\
\hline Bihar & 0 & 29 & 8 & 15 & 16 & 6 & 22 & 10 & 5 & 37 \\
\hline Chandigarh & 1 & 0 & 0 & 1 & 0 & 0 & 1 & 0 & 0 & 1 \\
\hline Chhattisgarh & 0 & 10 & 6 & 1 & 10 & 5 & 1 & 10 & 5 & 16 \\
\hline Dadra \& Nagar Haveli & 1 & 0 & 0 & 1 & 0 & 0 & 1 & 0 & 0 & 1 \\
\hline Daman \& Diu & 2 & 0 & 0 & 2 & 0 & 0 & 2 & 0 & 0 & 2 \\
\hline NCT Delhi & 0 & 9 & 0 & 0 & 9 & 0 & 0 & 9 & 0 & 9 \\
\hline Goa & 0 & 2 & 0 & 0 & 2 & 0 & 2 & 0 & 0 & 2 \\
\hline Gujarat & 7 & 4 & 14 & 7 & 4 & 14 & 7 & 4 & 14 & 25 \\
\hline Haryana & 0 & 0 & 19 & 1 & 0 & 18 & 4 & 2 & 13 & 19 \\
\hline Himachal Pradesh & 4 & 3 & 5 & 10 & 0 & 2 & 12 & 0 & 0 & 12 \\
\hline Jammu \& Kashmir & 3 & 4 & 7 & 14 & 0 & 0 & 14 & 0 & 0 & 14 \\
\hline Jharkhand & 0 & 12 & 6 & 0 & 15 & 3 & 8 & 7 & 3 & 18 \\
\hline Karnataka & 8 & 13 & 6 & 8 & 13 & 6 & 12 & 11 & 4 & 27 \\
\hline Kerala & 1 & 0 & 13 & 6 & 2 & 6 & 12 & 0 & 2 & 14 \\
\hline Lakshadweep & 1 & 0 & 0 & 1 & 0 & 0 & 1 & 0 & 0 & 1 \\
\hline Madhya Pradesh & 27 & 12 & 6 & 29 & 14 & 2 & 29 & 14 & 2 & 45 \\
\hline Maharashtra & 12 & 8 & 15 & 12 & 8 & 15 & 25 & 10 & 0 & 35 \\
\hline Manipur & 1 & 0 & 8 & 3 & 0 & 6 & 7 & 2 & 0 & 9 \\
\hline Meghalaya & 0 & 3 & 4 & 2 & 2 & 3 & 2 & 2 & 3 & 7 \\
\hline Mizoram & 0 & 8 & 0 & 1 & 7 & 0 & 1 & 7 & 0 & 8 \\
\hline Nagaland & 0 & 0 & 8 & 1 & 0 & 7 & 6 & 2 & 0 & 8 \\
\hline Orissa & 3 & 27 & 0 & 3 & 27 & 0 & 3 & 27 & 0 & 30 \\
\hline Pondicherry & 4 & 0 & 0 & 4 & 0 & 0 & 4 & 0 & 0 & 4 \\
\hline Punjab & 1 & 0 & 16 & 1 & 2 & 14 & 1 & 2 & 14 & 17 \\
\hline Rajasthan & 14 & 4 & 14 & 18 & 6 & 8 & 22 & 6 & 4 & 32 \\
\hline Sikkim & 2 & 0 & 2 & 4 & 0 & 0 & 4 & 0 & 0 & 4 \\
\hline Tamil Nadu & 3 & 13 & 14 & 7 & 20 & 3 & 13 & 15 & 2 & 30 \\
\hline Tripura & 0 & 0 & 4 & 1 & 0 & 3 & 1 & 0 & 3 & 4 \\
\hline Uttar Pradesh & 15 & 25 & 30 & 17 & 25 & 28 & 28 & 21 & 21 & 70 \\
\hline Uttaranchal & 2 & 0 & 11 & 2 & 2 & 9 & 3 & 2 & 8 & 13 \\
\hline West Bengal & 11 & 2 & 5 & 13 & 2 & 3 & 16 & 2 & 0 & 18 \\
\hline India & 136 & 204 & 249 & 211 & 199 & 183 & 317 & 169 & 107 & 593 \\
\hline
\end{tabular}




\section{Table 4. Districts with Boundaries Unchanged Between 1971 and 2001}

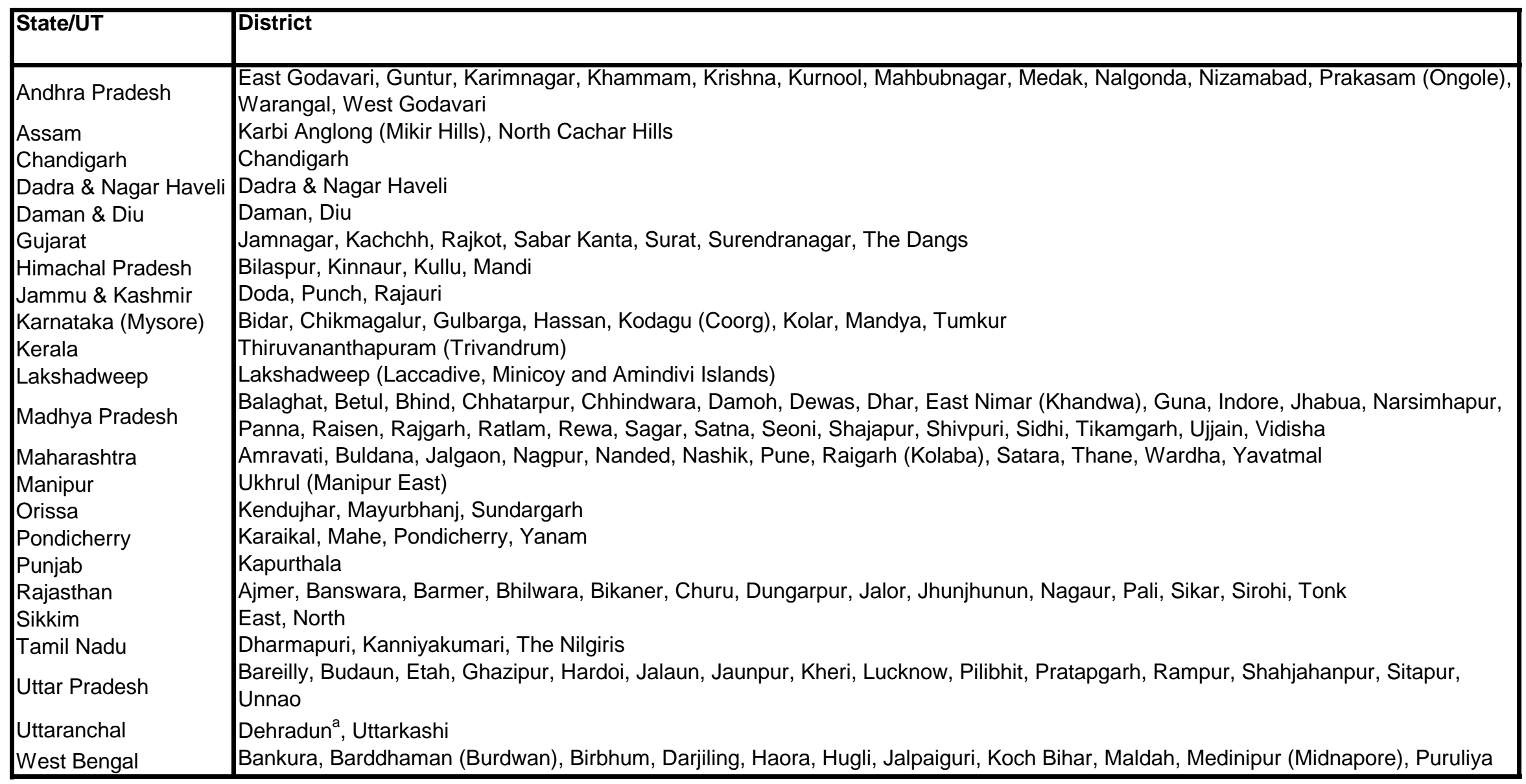

a One village with area 1 sq. km. and unknown population was transferred from Tehri Garhwal district to Dehradun district between 1971 and 1981.

Notes:

1. Districts are listed according to the state they were part of in 2001.

2. Where they are significantly different, older names of states and districts are mentioned in brackets. 


\begin{tabular}{|c|c|c|c|c|c|c|c|c|}
\hline $\begin{array}{c}\text { Census } \\
2001 \\
\text { State/UT }\end{array}$ & 1971 District & $\begin{array}{l}\text { Population } \\
\text { in } 1971\end{array}$ & 1981 District & $\begin{array}{c}\text { Share of } \\
1971 \\
\text { District }\end{array}$ & 1991 District & $\begin{array}{c}\text { Share of } \\
1981 \\
\text { District }\end{array}$ & 2001 District & $\begin{array}{c}\text { Share of } \\
1991 \\
\text { District }\end{array}$ \\
\hline$[1]$ & [2] & [3] & [4] & [5] & [6] & [7] & [8] & [9] \\
\hline \multirow{2}{*}{$\begin{array}{l}\text { Andaman \& } \\
\text { Nicobar Is. }\end{array}$} & \multirow{2}{*}{ Andaman \& Nicobar Islands } & \multirow{2}{*}{115,133} & Andaman & 81.18 & Andamans & 100.00 & Andamans & 100.00 \\
\hline & & & Nicobar & 18.82 & Nicobars & 100.00 & Nicobars & 100.00 \\
\hline \multirow{7}{*}{$\begin{array}{l}\text { Arunachal } \\
\text { Pradesh }\end{array}$} & \multirow{3}{*}{ Kameng } & \multirow{3}{*}{86,001} & West Kameng & 58.44 & Tawang & 34.34 & Tawang & 100.00 \\
\hline & & & vest raming & & West Kameng & 65.66 & West Kameng & 100.00 \\
\hline & & & East Kameng & 41.56 & East Kameng & 100.00 & East Kameng & 100.00 \\
\hline & \multirow{2}{*}{ Lohit } & \multirow{2}{*}{62,865} & Dibang Valley & 24.23 & Dibang Valley & 100.00 & Dibang Valley & 100.00 \\
\hline & & & Lohit & 75.77 & Lohit & 100.00 & Lohit & 100.00 \\
\hline & \multirow{2}{*}{ Tirap } & \multirow{2}{*}{97,470} & \multirow{2}{*}{ Tirap } & \multirow{2}{*}{100.00} & Changlang & 48.36 & Changlang & 100.00 \\
\hline & & & & & Tirap & 51.64 & Tirap & 100.00 \\
\hline \multirow{7}{*}{ Assam ${ }^{a}$} & \multirow{4}{*}{ Lakhimpur } & \multirow{4}{*}{$2,122,719$} & \multirow{2}{*}{ Lakhimpur } & \multirow{2}{*}{ NA } & Lakhimpur & 22.65 & Lakhimpur & 100.00 \\
\hline & & & & & Dhemaji & 10.87 & Dhemaji & 100.00 \\
\hline & & & \multirow{2}{*}{ Dibrugarh } & \multirow{2}{*}{$\mathrm{NA}$} & Dibrugarh & 35.64 & Dibrugarh & 100.00 \\
\hline & & & & & Tinsukia & 30.83 & Tinsukia & 100.00 \\
\hline & \multirow{3}{*}{ Cachar } & \multirow{3}{*}{$1,713,318$} & & & Karimganj & 33.98 & Karimganj & 100.00 \\
\hline & & & Cachar & 100.00 & Hailakandi & 17.96 & Hailakandi & 100.00 \\
\hline & & & & & Cachar & 48.07 & Cachar & 100.00 \\
\hline & & & Nawada & 20.05 & Nawada & 100.00 & Nawada & 100.00 \\
\hline & Gaya & $4,457,473$ & Gaya & 57.15 & Jehanabad & 31.39 & Jehanabad & 100.00 \\
\hline & Gayd & $4,451,4 / 0$ & Gayd & 01.10 & Gaya & 68.61 & Gaya & 100.00 \\
\hline & & & Aurangabad & 22.80 & Aurangabad & 100.00 & Aurangabad & 100.00 \\
\hline & & & Rohtas & 49.35 & Rohtas & 100.00 & Kaimur (Bhabua) & 33.90 \\
\hline & Shahabad & $3,939,034$ & & & & 100.00 & Rohtas & 66.10 \\
\hline & & & Bhojpur & 50.65 & Bhojpur & 100.00 & Bhojpur & 62.24 \\
\hline & & & 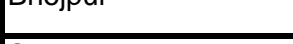 & 00.00 & 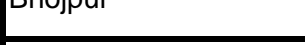 & 100.00 & Buxar & 37.76 \\
\hline & & & Saran & 39.95 & Saran & 100.00 & Saran & 100.00 \\
\hline & Saran & $4,279,253$ & Siwan & 34.17 & Siwan & 100.00 & Siwan & 100.00 \\
\hline & & & Gopalganj & 25.88 & Gopalganj & 100.00 & Gopalganj & 100.00 \\
\hline & Chamnaran & 3543,103 & Paschim Champaran & 44.79 & Pashchim Champaran & 100.00 & Pashchim Champaran & 100.00 \\
\hline & 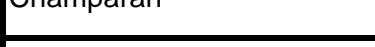 & $3,040,100$ & Purba Champaran & 55.21 & Purba Champaran & 100.00 & Purba Champaran & 100.00 \\
\hline & & & Sitamarhi & 32.69 & Sitamarhi & 100.00 & Sheohar & 15.79 \\
\hline Bihar & Muzaffarpur & $4,840,681$ & 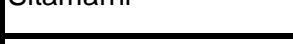 & & & & Sitamarhi & 84.21 \\
\hline & & & Muzaffarpur & 39.44 & Muzaffarpur & 100.00 & Muzaffarpur & 100.00 \\
\hline & & & Vaishali & 27.87 & Vaishali & 100.00 & Vaishali & 100.00 \\
\hline & & & Samastipur & 32.84 & Samastipur & 100.00 & Samastipur & 100.00 \\
\hline & Darbhanga & $5,233,904$ & Darbhanga & 31.01 & Darbhanga & 100.00 & Darbhanga & 100.00 \\
\hline & & & Madhubani & 36.15 & Madhubani & 100.00 & Madhubani & 100.00 \\
\hline & Bhagalpur & $2,091,103$ & Bhagalpur & 100.00 & Bhagalpur & 100.00 & Bhagalpur & 59.64 \\
\hline & 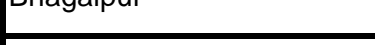 & $2,001,100$ & 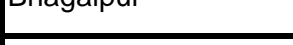 & 100.00 & Dilayalpuir & 100.00 & Banka & 40.36 \\
\hline & & & & & Saharsa & 67.36 & Supaul & 54.25 \\
\hline & Saharsa & $2,350,268$ & Saharsa & 100.00 & & 01.00 & Saharsa & 45.75 \\
\hline & & & & & Madhepura & 32.64 & Madhepura & 100.00 \\
\hline & & & & & Purnia & 42.22 & Purnia & 100.00 \\
\hline & Purnea & 3941863 & Purnia & 71.16 & Araria & 35.38 & Araria & 100.00 \\
\hline & r untea & $0,541,000$ & & & Kishanganj & 22.40 & Kishanganj & 100.00 \\
\hline & & & Katihar & 28.84 & Katihar & 100.00 & Katihar & 100.00 \\
\hline
\end{tabular}




\begin{tabular}{|c|c|c|c|c|c|c|c|c|}
\hline $\begin{array}{c}\text { Census } \\
2001 \\
\text { State/UT }\end{array}$ & 1971 District & $\begin{array}{c}\text { Population } \\
\text { in } 1971\end{array}$ & 1981 District & \begin{tabular}{|c} 
Share of \\
1971 \\
District
\end{tabular} & 1991 District & $\begin{array}{c}\text { Share of } \\
1981 \\
\text { District }\end{array}$ & 2001 District & $\begin{array}{c}\text { Share of } \\
1991 \\
\text { District }\end{array}$ \\
\hline [1] & [2] & [3] & [4] & [5] & [6] & [7] & [8] & [9] \\
\hline \multirow{9}{*}{ Chhattisgarh } & Surguja & $1,326,439$ & Surguja & 100.00 & Sarguja & 100.00 & Koriya & $\frac{24.04}{7506}$ \\
\hline & & & & & & & Jashpur & 38.11 \\
\hline & Ralgarn & I,2/8,/05 & Ralgarn & 100.00 & Ralgarn & 100.00 & Raigarh & 61.89 \\
\hline & \multirow{3}{*}{ Raipur } & \multirow{3}{*}{$2,613,531$} & \multirow{3}{*}{ Raipur } & \multirow{3}{*}{100.00} & \multirow{3}{*}{ Raipur } & \multirow{3}{*}{100.00} & Raipur & 64.72 \\
\hline & & & & & & & Mahasamund & 20.25 \\
\hline & & & & & & & Dhamtari & 15.04 \\
\hline & \multirow{3}{*}{ Bastar } & \multirow{3}{*}{$1,515,956$} & \multirow{3}{*}{ Bastar } & \multirow{3}{*}{100.00} & \multirow{3}{*}{ Bastar } & \multirow{3}{*}{100.00} & Kanker & 24.15 \\
\hline & & & & & & & Bastar & 48.46 \\
\hline & & & & & & & Dantewada & 27.40 \\
\hline \multirow{9}{*}{ Delhi } & \multirow{9}{*}{ Delhi } & \multirow{9}{*}{$4,065,698$} & \multirow{9}{*}{ Delhi } & \multirow{9}{*}{100.00} & \multirow{9}{*}{ Delhi } & \multirow{9}{*}{100.00} & North West & 18.87 \\
\hline & & & & & & & North & 7.29 \\
\hline & & & & & & & North East & 11.52 \\
\hline & & & & & & & East & 10.86 \\
\hline & & & & & & & New Delhi & 1.79 \\
\hline & & & & & & & Central & 6.97 \\
\hline & & & & & & & West & 15.21 \\
\hline & & & & & & & South West & 11.54 \\
\hline & & & & & & & South & 15.94 \\
\hline \multirow{2}{*}{ Goa } & \multirow{2}{*}{ Goa } & 795.120 & Goa & 10000 & North Goa & 56.37 & North Goa & 100.00 \\
\hline & & & & & South Goa & 43.63 & South Goa & 100.00 \\
\hline & Panch Mahals & $1,848,804$ & Panch Mahals & 100.00 & Panch Mahals & 100.00 & Panch Mahals & 56.90 \\
\hline Gujarat & & & & & & & Dohad & 43.10 \\
\hline & Valsad (Bulsar) & 1.428 .742 & Valsad & 100.00 & Valsad & 10000 & Navsari & 49.95 \\
\hline & & & & & valsau & & Valsad & 50.05 \\
\hline Himachal & & & Kangra & 60.34 & Kangra & 100.00 & Kangra & 100.00 \\
\hline Pradesh & Kangra & $1,327,211$ & Hamirpur & 19.97 & Hamirpur & 100.00 & Hamirpur & 100.00 \\
\hline & & & Una & 19.69 & Una & 100.00 & Una & 100.00 \\
\hline & Anantnag & 832280 & Anantnag (Kashmir South) & 62.25 & Anantnag & 100.00 & Anantnag & 100.00 \\
\hline Jammu \& & Anantnag & 832,280 & Pulwama & 37.75 & Pulwama & 100.00 & Pulwama & 100.00 \\
\hline Kashmir $^{\mathrm{a}}$ & adakh & 105291 & Kargil & 50.72 & Kargil & 100.00 & Kargil & 100.00 \\
\hline & & & Ladakh (Leh) & 49.28 & Leh (Ladakh) & 100.00 & Leh (Ladakh) & 100.00 \\
\hline & & & & & Godda & 19.19 & Godda & 100.00 \\
\hline & & & & & Sahibqani & 29.04 & Sahibganj & 56.63 \\
\hline & Santal Parganas & $3,186,908$ & Santhal Pargana & 100.00 & Salingalit & & Pakaur & 43.37 \\
\hline & & & & & Dumka & 32.70 & Dumka & 100.00 \\
\hline & & & & & Deoghar & 19.07 & Deoghar & 100.00 \\
\hline Jharkhand & Palamau & 1504350 & Palamu & 10000 & Palamu & 10000 & Garhwa & 32.69 \\
\hline & & & & & & & Palamu & 67.31 \\
\hline & & & & & Lohardaga & 7.48 & Lohardaga & 100.00 \\
\hline & Ranchi & $2,611,445$ & Ranchi & 100.00 & Gumla & 33.13 & Gumla & 100.00 \\
\hline & & & & & Ranchi & 59.39 & Ranchi & 100.00 \\
\hline & Singhbhum & 2437799 & Singhbhum & 10000 & Purbi Singhbhum & 48.18 & Purbi Singhbhum & 100.00 \\
\hline & 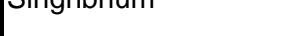 & ט & 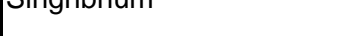 & 100.00 & Pashchimi Singhbhum & 51.82 & Pashchimi Singhbhum & 100.00 \\
\hline
\end{tabular}




\begin{tabular}{|c|c|c|c|c|c|c|c|c|}
\hline $\begin{array}{c}\text { Census } \\
2001 \\
\text { State/UT }\end{array}$ & 1971 District & $\begin{array}{c}\text { Population } \\
\text { in } 1971\end{array}$ & 1981 District & $\begin{array}{c}\text { Share of } \\
1971 \\
\text { District }\end{array}$ & 1991 District & $\begin{array}{c}\text { Share of } \\
1981 \\
\text { District }\end{array}$ & 2001 District & $\begin{array}{c}\text { Share of } \\
1991 \\
\text { District }\end{array}$ \\
\hline [1] & [2] & [3] & [4] & [5] & [6] & [7] & [8] & [9] \\
\hline \multirow{13}{*}{ Karnataka } & \multirow{2}{*}{ Bangalore } & \multirow{2}{*}{$3,365,515$} & \multirow{2}{*}{ Bangalore } & \multirow{2}{*}{100.00} & Bangalore & 70.65 & Bangalore & 100.00 \\
\hline & & & & & Bangalore Rural & 29.35 & Bangalore Rural & 100.00 \\
\hline & \multirow[t]{2}{*}{ Bijapur } & \multirow[t]{2}{*}{$1,985,591$} & \multirow[t]{2}{*}{ Bijapur } & \multirow[t]{2}{*}{100.00} & \multirow[t]{2}{*}{ Bijapur } & \multirow[t]{2}{*}{100.00} & Bagalkot & 47.48 \\
\hline & & & & & & & Bijapur & 52.52 \\
\hline & \multirow{3}{*}{ Dharwar } & \multirow{3}{*}{$2,342,213$} & \multirow{3}{*}{ Dharwad } & \multirow{3}{*}{100.00} & \multirow{3}{*}{ Dharwad } & \multirow{3}{*}{100.00} & Gadag & 24.52 \\
\hline & & & & & & & Dharwad & 39.25 \\
\hline & & & & & & & Haveri & 36.23 \\
\hline & \multirow{2}{*}{ Mysore District } & \multirow{2}{*}{$2,077,238$} & \multirow{2}{*}{ Mysore } & \multirow{2}{*}{100.00} & \multirow{2}{*}{ Mysore } & 100.00 & Mysore & 72.09 \\
\hline & & & & & & & Chamarajanagar & 27.91 \\
\hline & Raichur & $1,415,740$ & Raichur & 100.00 & Raichur & 100.00 & Raichur & 58.52 \\
\hline & & & & & & 100.00 & Koppal & 41.48 \\
\hline & South Kanara & $1,939,315$ & Dakshin Kannad & 100.00 & Dakshin Kannad & 100.00 & Udupi & 38.53 \\
\hline & Juntir Taliala & טונט,כט, ו & Lansintir Tariliau & & 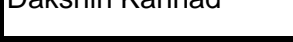 & & Dakshina Kannada & 61.47 \\
\hline & Morena & 985,338 & Morena & 100.00 & Morena & 100.00 & Sheopur & 25.22 \\
\hline & & & & & & & Morena & 74.78 \\
\hline & Shahdol & $1,029,839$ & Shahdol & 100.00 & Shahdol & 100.00 & Umaria & 24.13 \\
\hline & & $1,0<9,03 \checkmark$ & STaniút & 100.00 & Stranum & & Shahdol & 75.87 \\
\hline & Mandsaur & 961.522 & Mandsaur & 100.00 & Mandsaur & 100.00 & Neemuch & 38.47 \\
\hline Madhya & & & & & & & Mandsaur & 61.53 \\
\hline Pradesh & Kharaone (West Nimar) & 1.284 .812 & West Nimar & 10000 & West Nimar & 10000 & West Nimar & 58.96 \\
\hline & & $1, \angle 04,01<$ & & 100.00 & vost TuImal & 100.00 & Barwani & 41.04 \\
\hline & Jabalour & 1686.030 & Jabalpur & 100.00 & Jabalpur & 100.00 & Katni & 33.28 \\
\hline & & & & & & 100.00 & Jabalpur & 66.72 \\
\hline & Mandla & 873.577 & Mandla & 100.00 & Mandla & 100.00 & Dindori & 39.64 \\
\hline & IVranara & $8 / 3,5 / 1$ & IVianiara & 100.00 & IVIariuia & 100.00 & Mandla & 60.36 \\
\hline & Greater Bombay & 5970575 & Greater Bombay & 100.00 & Greater Bombay & 10000 & Mumbai (Suburban) & 68.01 \\
\hline & & & & & & & Mumbai & 31.99 \\
\hline & Dhulia & $1,662,181$ & Dhule & 100.00 & Dhule & 100.00 & Nandurbar & 41.90 \\
\hline Maharashtra & & & & 100.00 & Driule & 100.00 & Dhule & 58.10 \\
\hline & Akola & $1,501,478$ & Akola & $10000-2-3-2 \cdot$ & Akola & 10000 & Akola & 61.06 \\
\hline & & & & 100.00 & & & Washim & 38.94 \\
\hline & Bhandara & $1,585,580$ & Bhandara & 100.00 & Bhandara & 100.00 & Bhandara & 48.46 \\
\hline & & & & & & & Gondiya & 51.54 \\
\hline & & & East Garo Hills & 25.26 & East Garo Hills & 100.00 & East Garo Hills & 100.00 \\
\hline Meghalaya & Garo Hills & 406,615 & West Garn Hills & 7474 & West Garn Hills & 10000 & West Garo Hills & 83.95 \\
\hline & & & West Garo Hills & 74./4 & West Garo Hills & 100.00 & South Garo Hills & 16.05 \\
\hline & & & & & & & Mamit & 13.50 \\
\hline & & & & & & & Kolasib & 10.19 \\
\hline & & & Aizawl & 68.93 & Aizawl & 100.00 & Aizawl & 49.30 \\
\hline Mizoram & Mizo & 332390 & & & & & Champhai & 17.44 \\
\hline |VIILOCIAm & $|V||| \angle O$ & 332,390 & & & & & Serchhip & 9.57 \\
\hline & & & Lunglei & 18.69 & Lunglei & 100.00 & Lunglei & 100.00 \\
\hline & & & Chhimtuipui & 12.38 & Chhimtuipui & 100.00 & Lawngtlai & 54.27 \\
\hline & & & & & & 100.00 & Saiha & 45.73 \\
\hline
\end{tabular}




\begin{tabular}{|c|c|c|c|c|c|c|c|c|}
\hline $\begin{array}{c}\text { Census } \\
2001 \\
\text { State/UT }\end{array}$ & 1971 District & $\begin{array}{c}\text { Population } \\
\text { in } 1971\end{array}$ & 1981 District & $\begin{array}{c}\text { Share of } \\
1971 \\
\text { District }\end{array}$ & 1991 District & $\begin{array}{c}\text { Share of } \\
1981 \\
\text { District }\end{array}$ & 2001 District & $\begin{array}{c}\text { Share of } \\
1991 \\
\text { District }\end{array}$ \\
\hline [1] & {$[2]$} & [3] & [4] & [5] & [6] & {$[7]$} & [8] & [9] \\
\hline \multirow{27}{*}{ Orissa } & \multirow{4}{*}{ Sambalpur } & \multirow{4}{*}{$1,844,898$} & \multirow{4}{*}{ Sambalpur } & \multirow{4}{*}{100.00} & \multirow{4}{*}{ Sambalpur } & \multirow{4}{*}{100.00} & Bargarh & 44.76 \\
\hline & & & & & & & Jharsuguda & 16.39 \\
\hline & & & & & & & Sambalpur & 30.16 \\
\hline & & & & & & & Debagarh & 8.68 \\
\hline & Balasore & 1.830 .504 & Baleshwar & 100.00 & Baleshwar & 100.00 & Baleshwar & 60.54 \\
\hline & & & & & & & Bhadrak & 39.46 \\
\hline & \multirow{4}{*}{ Cuttack } & \multirow{4}{*}{$3,827,678$} & \multirow{4}{*}{ Cuttack } & \multirow{4}{*}{100.00} & \multirow{4}{*}{ Cuttack } & \multirow{4}{*}{100.00} & Kendrapara & 20.81 \\
\hline & & & & & & & Jagatsinghapur & 16.91 \\
\hline & & & & & & & Cuttack & 37.18 \\
\hline & & & & & & & Jajapur & 25.10 \\
\hline & \multirow{2}{*}{ Dhenkanal } & \multirow{2}{*}{$1,293,914$} & \multirow{2}{*}{ Dhenkanal } & \multirow{2}{*}{100.00} & \multirow{2}{*}{ Dhenkanal } & \multirow{2}{*}{100.00} & Dhenkanal & 49.66 \\
\hline & & & & & & & Anugul & 50.34 \\
\hline & \multirow{2}{*}{ Baudh Khondmals } & \multirow{2}{*}{621,675} & \multirow{2}{*}{ Phulabani } & 100.00 & Phulbani & 10000 & Kandhamal & 63.23 \\
\hline & & & & 100.00 & 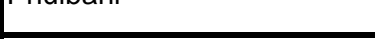 & & Baudh & 36.77 \\
\hline & Bolangir & $1,263,657$ & Balangir & 100.00 & Balangir & 100.00 & Sonapur & 27.92 \\
\hline & & & & & & & Balangir & 72.08 \\
\hline & Kalahandi & $1,163,869$ & Kalahandi & 100.00 & Kalahandi & 100.00 & Nuapada & 29.34 \\
\hline & 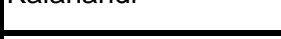 & & & & 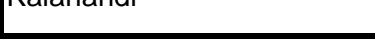 & 100.00 & Kalahandi & 70.66 \\
\hline & & & & & & & Rayagada & 23.70 \\
\hline & Koranut & 2043281 & Koranut & 10000 & Koranut & 10000 & Nabarangapur & 28.10 \\
\hline & 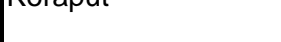 & 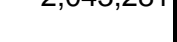 & 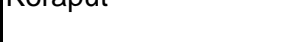 & 100.00 & титари & 100.00 & Koraput & 34.18 \\
\hline & & & & & & & Malkangiri & 14.02 \\
\hline & Ganiam & $2,293,808$ & Ganiam & 100.00 & Ganiam & 100.00 & Ganjam & 85.60 \\
\hline & & & & & & & Gajapati & 14.40 \\
\hline & & & & & & & Nayagarh & 21.80 \\
\hline & Puri & $2,340,859$ & Puri & 100.00 & Puri & 100.00 & Khordha & 41.84 \\
\hline & & & & & & & Puri & 36.36 \\
\hline & Ganganagar & $1,394,011$ & Ganganaqar & 10000 & Ganqanagar & 100.00 & Ganganagar & 53.47 \\
\hline Raiacthan & & & Mantyan ragai & 100.00 & mantyan iagai & & Hanumangarh & 46.53 \\
\hline 1 & Bharatour & 1490206 & Bharatpur & 10000 & Bharatpur & 68.95 & Bharatpur & 100.00 \\
\hline & 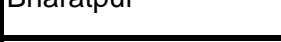 & , & 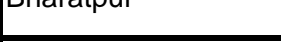 & & Dholpur & 31.05 & Dhaulpur & 100.00 \\
\hline & North Arcot & 3755.797 & North Arcot & 10000 & North Arcot-Ambedkar & 59.55 & Vellore & 100.00 \\
\hline & IV & ונה & IV & 100.00 & Tiruvannamalai-Sambuvara & 40.45 & Tiruvannamalai & 100.00 \\
\hline & South Arcot & 3617723 & South Arcot & 100.00 & South Arcot & 100.00 & Viluppuram & 56.49 \\
\hline & & & & & South Arcot & 100.00 & Cuddalore & 43.51 \\
\hline & Salem & $2,992,616$ & Salem & 100.00 & Salem & 100.00 & Salem & 66.05 \\
\hline & & & & 100.00 & valcin & 100.00 & Namakkal & 33.95 \\
\hline Tamil Nadu & Coimbatore & $4,373,178$ & Periyar & 41.09 & Periyar & 100.00 & Erode & 100.00 \\
\hline & & & Coimbatore & 58.91 & Coimbatore & 100.00 & Coimbatore & 100.00 \\
\hline & & & & & Dindigul Anna & 34.49 & Dindigul & 100.00 \\
\hline & Madurai & $3,938,197$ & Madurai & 100.00 & Madurai & 6551 & Madurai & 69.58 \\
\hline & & & & & IViacural & וJ. & Theni & 30.42 \\
\hline & Tirunelveli & 3200515 & Tirunelveli & 10000 & Chidambaranar & 37.79 & Thoothukkudi & 100.00 \\
\hline & & & & 100.00 & Tirunelveli-Kattabomman & 62.21 & Tirunelveli & 100.00 \\
\hline
\end{tabular}




\begin{tabular}{|c|c|c|c|c|c|c|c|c|}
\hline $\begin{array}{c}\text { Census } \\
2001 \\
\text { State/UT }\end{array}$ & 1971 District & $\begin{array}{l}\text { Population } \\
\text { in } 1971\end{array}$ & 1981 District & $\begin{array}{c}\text { Share of } \\
1971 \\
\text { District }\end{array}$ & 1991 District & $\begin{array}{c}\text { Share of } \\
1981 \\
\text { District }\end{array}$ & 2001 District & $\begin{array}{c}\text { Share of } \\
1991 \\
\text { District }\end{array}$ \\
\hline$[1]$ & [2] & [3] & [4] & [5] & [6] & [7] & [8] & [9] \\
\hline \multirow{19}{*}{$\begin{array}{l}\text { Uttar } \\
\text { Pradesh }\end{array}$} & Moradabad & $2,428,971$ & Moradabad & 100.00 & Moradabad & 100.00 & $\begin{array}{l}\text { Moradabad } \\
\text { Jyotiba Phule Nagar }\end{array}$ & $\frac{71.96}{28.04}$ \\
\hline & Farrukhabad & $1,556,930$ & Farrukhabad & 100.00 & Farrukhabad & 100.00 & \begin{tabular}{|l} 
Farrukhabad \\
Kannauj
\end{tabular} & $\begin{array}{l}52.63 \\
47.37\end{array}$ \\
\hline & Allahabad & $2,937,278$ & Allahabad & 100.00 & Allahabad & 100.00 & \begin{tabular}{|l|} 
Kaushambi \\
Allahabad \\
\end{tabular} & $\begin{array}{l}20.78 \\
79.22 \\
\end{array}$ \\
\hline & \multirow{2}{*}{ Jhansi } & \multirow{2}{*}{$1,307,058$} & Jhansi & 66.57 & Jhansi & 100.00 & Jhansi & 100.00 \\
\hline & & & Lalitpur & 33.43 & Lalitpur & 100.00 & Lalitpur & 100.00 \\
\hline & Hamirpur & 988,215 & Hamirpur & 100.00 & Hamirpur & 100.00 & $\begin{array}{l}\text { Hamirpur } \\
\text { Mahoba }\end{array}$ & $\begin{array}{l}60.31 \\
39.69\end{array}$ \\
\hline & Banda & $1,182,215$ & Banda & 100.00 & Banda & 100.00 & $\begin{array}{l}\text { Banda } \\
\text { Chitrakoot }\end{array}$ & $\frac{67.99}{32.01}$ \\
\hline & \multirow[t]{2}{*}{ Bahraich } & \multirow[t]{2}{*}{$1,726,972$} & \multirow[t]{2}{*}{ Bahraich } & \multirow[t]{2}{*}{100.00} & \multirow[t]{2}{*}{ Bahraich } & \multirow[t]{2}{*}{100.00} & Bahraich & 66.59 \\
\hline & & & & & & & Shrawasti & 33.41 \\
\hline & Gonda & $2,302,029$ & Gonda & 100.00 & Gonda & 100.00 & Balrampur & $\frac{38.30}{61.70}$ \\
\hline & \multirow{2}{*}{ Gorakhpur } & \multirow{2}{*}{$3,038,177$} & \multirow{2}{*}{ Gorakhpur } & \multirow{2}{*}{100.00} & Maharajganj & 35.17 & Mahrajganj & 100.00 \\
\hline & & & & & Gorakhpur & 64.83 & Gorakhpur & 100.00 \\
\hline & \multirow{2}{*}{ Deoria } & \multirow{2}{*}{$2,812,350$} & \multirow{2}{*}{ Deoria } & \multirow{2}{*}{100.00} & \multirow{2}{*}{ Deoria } & \multirow{2}{*}{100.00} & Kushinagar & 50.82 \\
\hline & & & & & & & Deoria & 49.18 \\
\hline & \multirow{3}{*}{ Varanasi } & \multirow{3}{*}{$2,852,459$} & \multirow{3}{*}{ Varanasi } & \multirow{3}{*}{100.00} & \multirow{3}{*}{ Varanasi } & \multirow{3}{*}{100.00} & Chandauli & 26.23 \\
\hline & & & & & & & Varanasi & 51.60 \\
\hline & & & & & & & Sant Ravidas Nagar Bhadohi & 22.17 \\
\hline & Mirzapur & 1.541 .088 & Mirzapur & 100.00 & Mirzapur & 61.85 & Mirzapur & 100.00 \\
\hline & & & & & Sonbhadra & 38.15 & Sonbhadra & 100.00 \\
\hline West Bengal & West Dinajpur & $1,859,887$ & West Dinajpur & 100.00 & West Dinajpur & 100.00 & Uttar Dinajpur & $\frac{60.65}{30.25}$ \\
\hline
\end{tabular}

${ }^{a}$ Census operations were not carried out in Assam in 1981 and Jammu \& Kashmir in 1991. No jurisdictional changes occurred in Jammu \& Kashmir between 1981 and 2001. For Assam, the only jurisdictional change in 1971-81 was the partitioning of Lakhimpur to create Dibrugarh. However, we do not know the population shares involved in this process. Hence the weights in column [5] are reported as "NA". Census 1991 documents do allow us to obtain the population shares of the 1971 district of Lakhimpur that went to its four child districts in 1991, and these numbers have been reported in column [7]. 
Table 6a. Boundary Changes in "Other" Districts: 1971-1981

\begin{tabular}{|c|c|c|c|c|c|}
\hline $\begin{array}{l}\text { Census } 1971 \\
\text { State/UT }\end{array}$ & 1971 District & $\begin{array}{c}\text { Population in } \\
1971\end{array}$ & 1981 District & $\begin{array}{c}\text { Share of } \\
1981 \\
\text { District in } \\
1971 \\
\text { District }\end{array}$ & $\begin{array}{c}\text { Share of } \\
1971 \\
\text { District in } \\
1981 \\
\text { District }\end{array}$ \\
\hline$[1]$ & {$[2]$} & [3] & {$[4]$} & [5] & [6] \\
\hline \multirow{12}{*}{ Andhra Pradesh } & Adilabad & $1,288,348$ & Adilabad & 100.00 & 100.00 \\
\hline & Anantapur & $2,115,321$ & Anantapuram & 100.00 & 100.00 \\
\hline & \multirow{2}{*}{ Chittoor } & \multirow{2}{*}{$2,285,536$} & Chittoor & 99.21 & 100.00 \\
\hline & & & Nellore & 0.79 & 1.11 \\
\hline & Cuddapah & $1,577,267$ & Cuddapah & 100.00 & 100.00 \\
\hline & \multirow{2}{*}{ Hyderabad } & \multirow{2}{*}{$2,791,762$} & Hyderabad & 60.27 & 100.00 \\
\hline & & & Rangareddy & 39.73 & 100.00 \\
\hline & Nellore & $1,609,617$ & Nellore & 100.00 & 98.89 \\
\hline & \multirow{2}{*}{ Srikakulam } & \multirow{2}{*}{$2,589,991$} & Srikakulam & 68.34 & 100.00 \\
\hline & & & Vizianagaram & 31.66 & 51.59 \\
\hline & \multirow{2}{*}{ Visakhapatnam } & \multirow{2}{*}{$2,805,366$} & Vishakhapatanam & 72.57 & 100.00 \\
\hline & & & Vizianagaram & 27.43 & 48.41 \\
\hline \multirow{5}{*}{ Arunachal Pradesh } & \multirow{3}{*}{ Siang } & \multirow{3}{*}{121,936} & East Siang & 40.19 & 100.00 \\
\hline & & & Upper Subansiri & 11.23 & 42.76 \\
\hline & & & West Siang & 48.58 & 100.00 \\
\hline & \multirow{2}{*}{ Subansiri } & \multirow{2}{*}{99,239} & Lower Subansiri & 81.53 & 100.00 \\
\hline & & & Upper Subansiri & 18.47 & 57.24 \\
\hline \multirow{5}{*}{ Assam } & Darrang & $1,736,188$ & Darrang & 100.00 & 100.00 \\
\hline & Goalpara & $2,225,103$ & Goalpara & 100.00 & 100.00 \\
\hline & Kamrup & $2,854,183$ & Kamrup & 100.00 & 100.00 \\
\hline & Nowgong & $1,680,895$ & Nowgong & 100.00 & 100.00 \\
\hline & Sibsagar & $1,837,389$ & Sibsagar & 100.00 & 100.00 \\
\hline \multirow{7}{*}{ Bihar } & Dhanbad & $1,466,417$ & Dhanbad & 100.00 & 100.00 \\
\hline & \multirow{2}{*}{ Hazaribagh } & \multirow{2}{*}{$3,020,214$} & Giridih & 45.51 & 100.00 \\
\hline & & & Hazaribagh & 54.49 & 100.00 \\
\hline & \multirow{2}{*}{ Monghyr } & \multirow{2}{*}{$3,892,609$} & Begusarai & 29.48 & 100.00 \\
\hline & & & Munger & 70.52 & 100.00 \\
\hline & \multirow{2}{*}{ Patna } & \multirow{2}{*}{$3,556,945$} & Nalanda & 36.72 & 100.00 \\
\hline & & & Patna & 63.28 & 100.00 \\
\hline & Ahmadabad & $2,910,307$ & Ahmadabad & 100.00 & 100.00 \\
\hline & Amreli & 848,730 & Amreli & 100.00 & 100.00 \\
\hline & Banas Kantha & $1,265,383$ & Banas Kantha & 100.00 & 100.00 \\
\hline & Bharuch (Broach) & $1,109,601$ & Bharuch & 100.00 & 100.00 \\
\hline Guiarat & Bhavnagar & $1,405,285$ & Bhavnagar & 100.00 & 100.00 \\
\hline Gujarat & Gandhinagar & 200,642 & Gandhinagar & 100.00 & 100.00 \\
\hline & Junagadh & $1,656,677$ & Junagadh & 100.00 & 100.00 \\
\hline & Kheda (Kaira) & $2,451,387$ & Kheda & 100.00 & 100.00 \\
\hline & Mehsana & $2,092,468$ & Mahesana & 100.00 & 100.00 \\
\hline & Vadodara (Baroda) & $1,980,065$ & Vadodara & 100.00 & 100.00 \\
\hline & Ambala & $1,098,405$ & Ambala & 100.00 & 100.00 \\
\hline & & & Faridabad & 41.91 & 100.00 \\
\hline & Gurgaon & $1,707,369$ & Gurgaon & 38.46 & 100.00 \\
\hline Haryana & & & Mahendragarh & 19.63 & 43.69 \\
\hline maryalia & & & Bhiwani & 20.79 & 63.07 \\
\hline & Hisar & 2132948 & Hisar & 54.07 & 99.35 \\
\hline & & & Jind & 0.12 & 0.33 \\
\hline & & & Sirsa & 25.02 & 100.00 \\
\hline
\end{tabular}


Table 6a. Boundary Changes in "Other" Districts: 1971-1981

\begin{tabular}{|c|c|c|c|c|c|}
\hline $\begin{array}{c}\text { Census } 1971 \\
\text { State/UT }\end{array}$ & 1971 District & $\begin{array}{c}\text { Population in } \\
1971\end{array}$ & 1981 District & $\begin{array}{c}\text { Share of } \\
1981 \\
\text { District in } \\
1971 \\
\text { District }\end{array}$ & $\begin{array}{c}\text { Share of } \\
1971 \\
\text { District in } \\
1981 \\
\text { District }\end{array}$ \\
\hline [1] & [2] & [3] & [4] & [5] & [6] \\
\hline \multirow{9}{*}{ Haryana } & \multirow{2}{*}{ Jind } & \multirow{2}{*}{639,610} & Hisar & 1.18 & 0.65 \\
\hline & & & Jind & 98.82 & 82.67 \\
\hline & \multirow{3}{*}{ Karnal } & \multirow{3}{*}{$1,981,303$} & Jind & 6.56 & 17.00 \\
\hline & & & Karnal & 49.89 & 100.00 \\
\hline & & & Kurukshetra & 43.55 & 100.00 \\
\hline & \multirow{2}{*}{ Mahendragarh } & \multirow{2}{*}{691,639} & Bhiwani & 37.55 & 36.93 \\
\hline & & & Mahendragarh & 62.45 & 56.31 \\
\hline & \multirow{2}{*}{ Rohtak } & \multirow{2}{*}{$1,785,534$} & Rohtak & 61.52 & 100.00 \\
\hline & & & Sonipat & 38.48 & 100.00 \\
\hline \multirow{8}{*}{ Himachal Pradesh } & \multirow{2}{*}{ Chamba } & \multirow{2}{*}{255,233} & Chamba & 98.42 & 100.00 \\
\hline & & & Lahul And Spiti & 1.58 & 14.62 \\
\hline & Lahul \& Spiti & 23,538 & Lahul And Spiti & 100.00 & 85.38 \\
\hline & \multirow{2}{*}{ Mahasu } & \multirow{2}{*}{440,118} & Shimla & 79.03 & 82.85 \\
\hline & & & Solan & 20.97 & 38.87 \\
\hline & \multirow{2}{*}{ Simla } & \multirow{2}{*}{217,129} & Shimla & 33.16 & 17.15 \\
\hline & & & Solan & 66.84 & 61.13 \\
\hline & Sirmaur & 245,033 & Sirmaur & 100.00 & 100.00 \\
\hline \multirow{10}{*}{ Jammu \& Kashmir } & \multirow{3}{*}{ Baramula } & \multirow{3}{*}{775,724} & Baramula (Kashmir North) & 66.04 & 100.00 \\
\hline & & & Kupwara & 33.24 & 100.00 \\
\hline & & & Srinagar & 0.73 & 1.00 \\
\hline & \multirow{3}{*}{ Jammu } & \multirow{3}{*}{731,743} & Jammu & 99.05 & 100.00 \\
\hline & & & Kathua & 0.42 & 1.10 \\
\hline & & & Udhampur & 0.53 & 1.13 \\
\hline & Kathua & 274,671 & Kathua & 100.00 & 98.90 \\
\hline & \multirow{2}{*}{ Srinagar } & \multirow{2}{*}{827,697} & Badgam & 32.50 & 100.00 \\
\hline & & & Srinagar & 67.50 & 99.00 \\
\hline & Udhampur & 338,846 & Udhampur & 100.00 & 98.87 \\
\hline \multirow{14}{*}{ Kerala } & Alleppey & $2,125,722$ & Alleppey & 100.00 & 100.00 \\
\hline & \multirow{2}{*}{ Cannanore } & $2,365,164$ & Cannanore & 94.53 & 100.00 \\
\hline & & & Wayanad & 5.47 & 31.25 \\
\hline & Ernakulam & $2,383,178$ & Ernakulam & 90.79 & 100.00 \\
\hline & & $2,000,1 / 0$ & Idukki & 9.21 & 28.67 \\
\hline & Kottayam & $2,085,134$ & Idukki & 26.19 & 71.33 \\
\hline & rutianan & $2,000,134$ & Kottayam & 73.81 & 100.00 \\
\hline & Kozhikode & $2,106,249$ & Kozhikode & 86.49 & 100.00 \\
\hline & & $2,100,249$ & Wayanad & 13.51 & 68.75 \\
\hline & Mallapuram & 1.856 .362 & Malappuram & 99.9998 & 100.00 \\
\hline & & $1,050,30<$ & Palghat & 0.0002 & 0.0003 \\
\hline & Palghat & $1,685,342$ & Palghat & 100.00 & 99.9997 \\
\hline & Quilon & $2,412,821$ & Quilon & 100.00 & 100.00 \\
\hline & Trichur & $2,128,797$ & Trichur & 100.00 & 100.00 \\
\hline
\end{tabular}


Table 6a. Boundary Changes in "Other" Districts: 1971-1981

\begin{tabular}{|c|c|c|c|c|c|}
\hline $\begin{array}{c}\text { Census } 1971 \\
\text { State/UT }\end{array}$ & 1971 District & $\begin{array}{c}\text { Population in } \\
1971\end{array}$ & 1981 District & \begin{tabular}{|c|} 
Share of \\
1981 \\
District in \\
1971 \\
District \\
\end{tabular} & $\begin{array}{c}\text { Share of } \\
1971 \\
\text { District in } \\
1981 \\
\text { District } \\
\end{array}$ \\
\hline [1] & {$[2]$} & {$[3]$} & [4] & [5] & {$[6]$} \\
\hline \multirow{9}{*}{ Madhya Pradesh } & Bilaspur & $2,440,962$ & Bilaspur & 100.00 & 100.00 \\
\hline & Datia & 255,267 & Datia & 100.00 & 100.00 \\
\hline & \multirow{2}{*}{ Durg } & \multirow{2}{*}{$2,461,901$} & Durg & 59.67 & 100.00 \\
\hline & & & Raj Nandgaon & 40.33 & 100.00 \\
\hline & Gwalior & 858,005 & Gwalior & 100.00 & 100.00 \\
\hline & Hoshangabad & 805,870 & Hoshangabad & 100.00 & 99.77 \\
\hline & \multirow{3}{*}{ Sehore } & \multirow{3}{*}{$1,084,933$} & Bhopal & 52.74 & 100.00 \\
\hline & & & Hoshangabad & 0.17 & 0.23 \\
\hline & & & Sehore & 47.09 & 100.00 \\
\hline \multirow{10}{*}{ Maharashtra } & Ahmednagar & $2,269,117$ & Ahmadnagar & 100.00 & 100.00 \\
\hline & Aurangabad & $1,971,006$ & Aurangabad & 100.00 & 100.00 \\
\hline & Bhir & $1,286,121$ & Bid & 100.00 & 100.00 \\
\hline & Chandrapur & $1,640,137$ & Chandrapur & 100.00 & 100.00 \\
\hline & Kolhapur & $2,048,049$ & Kolhapur & 100.00 & 100.00 \\
\hline & Osmanabad & $1,896,687$ & Osmanabad & 100.00 & 100.00 \\
\hline & Parbhani & $1,506,771$ & Parbhani & 100.00 & 100.00 \\
\hline & Ratnagiri & $1,990,583$ & Ratnagiri & 100.00 & 100.00 \\
\hline & Sangli & $1,539,820$ & Sangli & 100.00 & 100.00 \\
\hline & Sholapur & $2,253,840$ & Solapur & 100.00 & 100.00 \\
\hline \multirow{7}{*}{ Manipur } & \multirow{4}{*}{ Manipur Central } & \multirow{4}{*}{763,260} & Manipur Central & 94.46 & 100.00 \\
\hline & & & Manipur South & 0.33 & 2.52 \\
\hline & & & Manipur West & 0.14 & 2.30 \\
\hline & & & Tengnoupal & 5.07 & 100.00 \\
\hline & Manipur North & 104,175 & Manipur North & 100.00 & 100.00 \\
\hline & Manipur South & 98,114 & Manipur South & 100.00 & 97.48 \\
\hline & Manipur West & 44,975 & Manipur West & 100.00 & 97.70 \\
\hline \multirow{3}{*}{ Meghalaya } & \multirow{3}{*}{ United Khasi \& Jaintia Hills } & \multirow{3}{*}{605,084} & East Khasi Hills & 62.91 & 100.00 \\
\hline & & & Jaintia Hills & 18.77 & 100.00 \\
\hline & & & West Khasi Hills & 18.32 & 100.00 \\
\hline \multirow{5}{*}{ Mysore } & Belgaum & $2,423,342$ & Belgaum & 100.00 & 100.00 \\
\hline & Bellary & $1,122,686$ & Bellary & 100.00 & 100.00 \\
\hline & Chitradurga & $1,397,456$ & Chitradurga & 100.00 & 100.00 \\
\hline & North Kanara & 849,105 & Uttar Kannad & 100.00 & 100.00 \\
\hline & Shimoga & $1,301,485$ & Shimoga & 100.00 & 100.00 \\
\hline \multirow{7}{*}{ Nagaland } & \multirow{2}{*}{ Kohima } & \multirow{2}{*}{175,204} & Kohima & 74.55 & 100.00 \\
\hline & & & Phek & 25.45 & 100.00 \\
\hline & \multirow{3}{*}{ Mokokchung } & \multirow{3}{*}{168,242} & Mokokchung & 49.25 & 100.00 \\
\hline & & & Wokha & 22.76 & 100.00 \\
\hline & & & Zunheboto & 27.99 & 100.00 \\
\hline & \multirow{2}{*}{ Tuensang } & \multirow{2}{*}{173,003} & Mon & 37.07 & 100.00 \\
\hline & & & Tuensang & 62.93 & 100.00 \\
\hline
\end{tabular}


Table 6a. Boundary Changes in "Other" Districts: 1971-1981

\begin{tabular}{|c|c|c|c|c|c|}
\hline $\begin{array}{c}\text { Census } 1971 \\
\text { State/UT }\end{array}$ & 1971 District & $\begin{array}{c}\text { Population in } \\
1971\end{array}$ & 1981 District & \begin{tabular}{|c|} 
Share of \\
1981 \\
District in \\
1971 \\
District \\
\end{tabular} & $\begin{array}{c}\text { Share of } \\
1971 \\
\text { District in } \\
1981 \\
\text { District } \\
\end{array}$ \\
\hline$[1]$ & {$[2]$} & {$[3]$} & [4] & [5] & {$[6]$} \\
\hline \multirow{17}{*}{ Punjab } & Amritsar & $1,835,500$ & Amritsar & 100.00 & 100.00 \\
\hline & \multirow{2}{*}{ Bhatinda } & \multirow{2}{*}{$1,318,134$} & Bathinda & 77.78 & 100.00 \\
\hline & & & Faridkot & 22.22 & 25.38 \\
\hline & \multirow{2}{*}{ Firozpur } & \multirow{2}{*}{$1,905,833$} & Faridkot & 45.17 & 74.62 \\
\hline & & & Firozpur & 54.83 & 100.00 \\
\hline & Gurdaspur $^{a}$ & $1,229,249$ & Gurdaspur & 100.00 & 99.98 \\
\hline & \multirow{4}{*}{ Hoshiarpur } & \multirow{4}{*}{$1,052,153$} & Gurdaspur & 0.02 & 0.02 \\
\hline & & & Hoshiarpur & 99.90 & 100.00 \\
\hline & & & Ludhiana & 0.06 & 0.04 \\
\hline & & & Rupnagar & 0.02 & 0.04 \\
\hline & Jullundur & $1,454,501$ & Jalandhar & 100.00 & 100.00 \\
\hline & Ludhiana & $1,419,421$ & Ludhiana & 100.00 & 99.96 \\
\hline & \multirow{2}{*}{ Patiala } & \multirow{2}{*}{$1,215,100$} & Patiala & 98.69 & 99.02 \\
\hline & & & Rupnagar & 1.31 & 2.90 \\
\hline & \multirow{2}{*}{ Ropar } & \multirow{2}{*}{545,005} & Patiala & 2.17 & 0.98 \\
\hline & & & Rupnagar & 97.83 & 97.06 \\
\hline & Sangrur & $1,146,650$ & Sangrur & 100.00 & 100.00 \\
\hline \multirow{14}{*}{ Rajasthan } & Alwar & $1,391,162$ & Alwar & 100.00 & 99.10 \\
\hline & Bundi & 449,021 & Bundi & 100.00 & 100.00 \\
\hline & Chittaurgarh & 944,981 & Chittaurgarh & 100.00 & 99.99 \\
\hline & \multirow{2}{*}{ Jaipur } & \multirow{2}{*}{$2,482,385$} & Alwar & 0.51 & 0.90 \\
\hline & & & Jaipur & 99.49 & 100.00 \\
\hline & Jaisalmer & 166,761 & Jaisalmer & 100.00 & 99.37 \\
\hline & Jhalawar & 622,001 & Jhalawar & 100.00 & 99.72 \\
\hline & \multirow{2}{*}{ Jodhpur } & \multirow{2}{*}{$1,152,712$} & Jaisalmer & 0.09 & 0.63 \\
\hline & & & Jodhpur & 99.91 & 100.00 \\
\hline & \multirow{2}{*}{ Kota } & \multirow{2}{*}{$1,143,870$} & Jhalawar & 0.15 & 0.28 \\
\hline & & & Kota & 99.85 & 100.00 \\
\hline & Sawai Madhopur & $1,193,528$ & Sawai Madhopur & 100.00 & 100.00 \\
\hline & \multirow{2}{*}{ Udaipur } & \multirow{2}{*}{$1,803,680$} & Chittaurgarh & 0.01 & 0.01 \\
\hline & & & Udaipur & 99.99 & 100.00 \\
\hline \multirow{3}{*}{ Sikkim } & South District & 52,423 & South District & 100.00 & 98.57 \\
\hline & \multirow{2}{*}{ West District } & 58785 & South District & 1.30 & 1.43 \\
\hline & & 50,105 & West District & 98.70 & 100.00 \\
\hline & IChinglenut & 2907599 & Chengalpattu & 96.22 & 100.00 \\
\hline & |cningleput & $2,907,599$ & Madras & 3.78 & 4.26 \\
\hline & Madras & $2,469,449$ & Madras & 100.00 & 95.74 \\
\hline Tamil Nadu & Ramanathapuram & $2,860,207$ & Ramanathapuram & 100.00 & 100.00 \\
\hline I amil Nadu & Thaniavur & 3810732 & Pudukkottai & 7.57 & 30.68 \\
\hline & Inanjavur & $3,840, / 32$ & Thanjavur & 92.43 & 100.00 \\
\hline & & & Pudukkottai & 17.06 & 69.32 \\
\hline & I Iruchırapallı & $3,848,816$ & Tiruchchirappalli & 82.94 & 100.00 \\
\hline & North Tripura & 405,009 & North Tripura & 100.00 & 99.01 \\
\hline Trinume & South Tripura & 399,728 & South Tripura & 100.00 & 100.00 \\
\hline fripura & West Trinura & 751605 & North Tripura & 0.54 & 0.99 \\
\hline & |Vwest Iripura & 151,605 & West Tripura & 99.46 & 100.00 \\
\hline
\end{tabular}


Table 6a. Boundary Changes in "Other" Districts: 1971-1981

\begin{tabular}{|c|c|c|c|c|c|}
\hline $\begin{array}{c}\text { Census } 1971 \\
\text { State/UT }\end{array}$ & 1971 District & $\begin{array}{c}\text { Population in } \\
1971\end{array}$ & 1981 District & \begin{tabular}{|c|} 
Share of \\
1981 \\
District in \\
1971 \\
District
\end{tabular} & $\begin{array}{c}\text { Share of } \\
1971 \\
\text { District in } \\
1981 \\
\text { District }\end{array}$ \\
\hline [1] & [2] & [3] & [4] & [5] & [6] \\
\hline \multirow{28}{*}{ Uttar Pradesh } & Agra & $2,308,638$ & Agra & 100.00 & 100.00 \\
\hline & Aligarh & $2,111,829$ & Aligarh & 100.00 & 100.00 \\
\hline & \multirow{2}{*}{ Almora } & \multirow{2}{*}{750,038} & Almora & 86.48 & 100.00 \\
\hline & & & Pithoragarh & 13.52 & 24.43 \\
\hline & Azamgarh & $2,857,484$ & Azamgarh & 100.00 & 100.00 \\
\hline & Ballia & $1,588,935$ & Ballia & 100.00 & 100.00 \\
\hline & Bara Banki & $1,635,593$ & Bara Banki & 100.00 & 100.00 \\
\hline & Basti & $2,984,090$ & Basti & 100.00 & 100.00 \\
\hline & Bijnor & $1,490,185$ & Bijnor & 100.00 & 100.00 \\
\hline & \multirow{2}{*}{ Bulandshahr } & \multirow{2}{*}{$2,073,343$} & Bulandshahr & 91.23 & 100.00 \\
\hline & & & Ghaziabad & 8.77 & 13.57 \\
\hline & Chamoli & 292,571 & Chamoli & 100.00 & 100.00 \\
\hline & Etawah & $1,447,702$ & Etawah & 100.00 & 100.00 \\
\hline & Faizabad & $1,927,281$ & Faizabad & 100.00 & 100.00 \\
\hline & Fatehpur & $1,278,254$ & Fatehpur & 100.00 & 100.00 \\
\hline & Garhwal & 553,028 & Garhwal & 100.00 & 100.00 \\
\hline & Kanpur & $2,996,232$ & Kanpur & 100.00 & 100.00 \\
\hline & Mainpuri & $1,445,534$ & Mainpuri & 100.00 & 100.00 \\
\hline & Mathura & $1,290,307$ & Mathura & 100.00 & 100.00 \\
\hline & \multirow{2}{*}{ Meerut } & \multirow{2}{*}{$3,366,953$} & Ghaziabad & 34.42 & 86.43 \\
\hline & & & Meerut & 65.58 & 100.00 \\
\hline & Muzaffarnagar & $1,802,289$ & Muzaffarnagar & 100.00 & 100.00 \\
\hline & Naini Tal & 790,080 & Naini Tal & 100.00 & 100.00 \\
\hline & Pithoragarh & 313,747 & Pithoragarh & 100.00 & 75.57 \\
\hline & Rae Bareli & $1,510,812$ & Rae Bareli & 100.00 & 100.00 \\
\hline & Saharanpur & $2,054,834$ & Saharanpur & 100.00 & 100.00 \\
\hline & Sultanpur & $1,642,928$ & Sultanpur & 100.00 & 100.00 \\
\hline & Tehri Garhwal & 397,385 & Tehri Garhwal & 100.00 & 100.00 \\
\hline \multirow{5}{*}{ West Bengal } & Calcutta & $3,148,746$ & Calcutta & 100.00 & 100.00 \\
\hline & Murshidabad & $2,940,204$ & Murshidabad & 100.00 & 99.78 \\
\hline & \multirow{2}{*}{ Nadia } & \multirow{2}{*}{$2,230,270$} & Murshidabad & 0.29 & 0.22 \\
\hline & & & Nadia & 99.71 & 100.00 \\
\hline & Twenty Four Parganas & $8,449,482$ & Twenty Four Parganas & 100.00 & 100.00 \\
\hline
\end{tabular}

${ }^{a}$ One uninhabited village with an area of $0.2 \mathrm{sq} . \mathrm{km}$. was transferred from Gurdaspur district to Hoshiarpur district in 1973. 
Table 6b. Boundary Changes in "Other" Districts: 1981-1991

\begin{tabular}{|c|c|c|c|c|c|}
\hline $\begin{array}{l}\text { Census } 1981 \\
\text { State/UT }\end{array}$ & 1981 District & $\begin{array}{c}\text { Population in } \\
1981\end{array}$ & 1991 District & $\begin{array}{c}\text { Share of } \\
1991 \\
\text { District in } \\
1981 \text { District }\end{array}$ & $\begin{array}{c}\text { Share of } \\
1981 \text { District } \\
\text { in } 1991 \\
\text { District }\end{array}$ \\
\hline [1] & [2] & [3] & [4] & {$[5]$} & [6] \\
\hline \multirow{12}{*}{ Andhra Pradesh } & Adilabad & $1,639,003$ & Adilabad & 100.00 & 99.92 \\
\hline & Anantapuram & $2,548,012$ & Anantapur & 100.00 & 99.89 \\
\hline & Chittoor & $2,737,316$ & Chittoor & 100.00 & 100.00 \\
\hline & \multirow{2}{*}{ Cuddapah } & \multirow{2}{*}{$1,933,304$} & Anantapur & 0.15 & 0.11 \\
\hline & & & Cuddapah & 99.85 & 100.00 \\
\hline & \multirow{2}{*}{ Hyderabad } & \multirow{2}{*}{$2,260,702$} & Hyderabad & 99.57 & 100.00 \\
\hline & & & Rangareddi & 0.43 & 0.61 \\
\hline & Nellore & $2,014,879$ & Nellore & 100.00 & 100.00 \\
\hline & Rangareddy & $1,582,062$ & Rangareddi & 100.00 & 99.39 \\
\hline & Srikakulam & $1,959,352$ & Srikakulam & 100.00 & 100.00 \\
\hline & Vishakhapatanam & $2,576,474$ & Visakhapatanam & 100.00 & 100.00 \\
\hline & Vizianagaram & $1,804,196$ & Vizianagaram & 100.00 & 100.00 \\
\hline \multirow{5}{*}{ Arunachal Pradesh } & East Siang & 70,451 & East Siang & 100.00 & 92.34 \\
\hline & Lower Subansiri & 112,650 & Lower Subansiri & 100.00 & 100.00 \\
\hline & Upper Subansiri & 39,410 & Upper Subansiri & 100.00 & 100.00 \\
\hline & \multirow{2}{*}{ West Siang } & \multirow{2}{*}{74,164} & East Siang & 7.88 & 7.66 \\
\hline & & & West Siang & 92.12 & 100.00 \\
\hline \multirow{17}{*}{ Assam } & \multirow{2}{*}{ Darrang } & \multirow{2}{*}{$1,736,188$} & Darrang & 48.07 & 100.00 \\
\hline & & & Sonitpur & 51.93 & 99.47 \\
\hline & \multirow{4}{*}{ Goalpara } & \multirow{4}{*}{$2,225,103$} & Bongaigaon & 21.91 & 99.42 \\
\hline & & & Dhubri & 38.25 & 100.00 \\
\hline & & & Goalpara & 19.48 & 100.00 \\
\hline & & & Kokrajhar & 20.35 & 100.00 \\
\hline & \multirow{4}{*}{ Kamrup } & \multirow{4}{*}{$2,854,183$} & Barpeta & 33.95 & 100.00 \\
\hline & & & Bongaigaon & 0.10 & 0.58 \\
\hline & & & Kamrup & 42.10 & 99.56 \\
\hline & & & Nalbari & 23.86 & 100.00 \\
\hline & \multirow{3}{*}{ Nowgong } & \multirow{3}{*}{$1,680,895$} & Kamrup & 0.32 & 0.44 \\
\hline & & & Marigaon & 25.22 & 100.00 \\
\hline & & & Nagaon & 74.46 & 100.00 \\
\hline & \multirow{4}{*}{ Sibsagar } & \multirow{4}{*}{$1,837,389$} & Golaghat & 28.50 & 100.00 \\
\hline & & & Jorhat & 35.62 & 100.00 \\
\hline & & & Sibsagar & 35.61 & 100.00 \\
\hline & & & Sonitpur & 0.26 & 0.53 \\
\hline & Begusarai & $1,456,343$ & Begusarai & 100.00 & 100.00 \\
\hline & Dhanbad & $2,115,010$ & Dhanbad & 100.00 & 100.00 \\
\hline & Giridih & $1,731,462$ & Giridih & 100.00 & 100.00 \\
\hline Rihar & Hazaribagh & $2,198,310$ & Hazaribag & 100.00 & 100.00 \\
\hline BInar & Munger & 3315407 & Khagaria & 23.18 & 100.00 \\
\hline & iviumger & $0,010,4<1$ & Munger & 76.82 & 100.00 \\
\hline & Nalanda & $1,641,325$ & Nalanda & 100.00 & 100.00 \\
\hline & Patna & $3,019,201$ & Patna & 100.00 & 100.00 \\
\hline & Ahmadabad $^{\mathrm{a}}$ & $3,875,794$ & Ahmadabad & 100.00 & 100.00 \\
\hline & Amreli & $1,079,097$ & Amreli & 100.00 & 100.00 \\
\hline & Banas Kantha & $1,667,914$ & Banas Kantha & 100.00 & 100.00 \\
\hline & Bharuch & $1,296,451$ & Bharuch & 100.00 & 100.00 \\
\hline Guiarat & Bhavnagar & $1,879,340$ & Bhavnagar & 100.00 & 100.00 \\
\hline Mujar at & Gandhinagar $^{\mathrm{a}}$ & 289,088 & Gandhinagar & 100.00 & 100.00 \\
\hline & Junagadh & $2,100,709$ & Junagadh & 100.00 & 100.00 \\
\hline & Kheda & $3,015,027$ & Kheda & 100.00 & 100.00 \\
\hline & Mahesana & $2,548,787$ & Mahasana & 100.00 & 100.00 \\
\hline & Vadodara & $2,558,092$ & Vadodara & 100.00 & 100.00 \\
\hline
\end{tabular}


Table 6b. Boundary Changes in "Other" Districts: 1981-1991

\begin{tabular}{|c|c|c|c|c|c|}
\hline $\begin{array}{l}\text { Census } 1981 \\
\text { State/UT }\end{array}$ & 1981 District & $\begin{array}{c}\text { Population in } \\
1981\end{array}$ & 1991 District & $\begin{array}{c}\text { Share of } \\
1991 \\
\text { District in } \\
1981 \text { District }\end{array}$ & \begin{tabular}{|c} 
Share of \\
1981 District \\
in 1991 \\
District
\end{tabular} \\
\hline$[1]$ & [2] & [3] & [4] & [5] & [6] \\
\hline \multirow{29}{*}{ Haryana $^{b}$} & Ambala & 1409463 & Ambala & 61.37 & 100.00 \\
\hline & Ambala & $1,409,463$ & Yamunanagar & 38.63 & 85.62 \\
\hline & Bhiwani & 920,052 & Bhiwani & 100.00 & 99.22 \\
\hline & \multirow{4}{*}{ Faridabad } & \multirow{4}{*}{$1,000,859$} & Bulandshahr & 0.03 & 0.01 \\
\hline & & & Faridabad & 98.41 & 99.89 \\
\hline & & & Ghaziabad & 0.02 & 0.01 \\
\hline & & & Gurgaon & 1.54 & 1.78 \\
\hline & \multirow{2}{*}{ Gurgaon } & \multirow{2}{*}{849,598} & Faridabad & 0.13 & 0.11 \\
\hline & & & Gurgaon & 99.87 & 98.22 \\
\hline & \multirow{2}{*}{ Hisar } & \multirow{2}{*}{$1,496,534$} & Hisar & 99.53 & 100.00 \\
\hline & & & Rohtak & 0.47 & 0.46 \\
\hline & \multirow{3}{*}{ Jind } & \multirow{3}{*}{938,074} & Jind & 83.40 & 100.00 \\
\hline & & & Kaithal & 14.08 & 20.47 \\
\hline & & & Panipat & 2.51 & 3.77 \\
\hline & \multirow{3}{*}{ Karnal } & \multirow{3}{*}{$1,322,826$} & Karnal & 52.85 & 99.88 \\
\hline & & & Kurukshetra & 1.67 & 4.05 \\
\hline & & & Panipat & 45.47 & 96.23 \\
\hline & \multirow{4}{*}{ Kurukshetra } & \multirow{4}{*}{$1,130,026$} & Kaithal & 45.42 & 79.53 \\
\hline & & & Karnal & 0.08 & 0.12 \\
\hline & & & Kurukshetra & 46.41 & 95.95 \\
\hline & & & Yamunanagar & 8.09 & 14.38 \\
\hline & \multirow{3}{*}{ Mahendragarh } & \multirow{3}{*}{959,400} & Bhiwani & 0.76 & 0.78 \\
\hline & & & Mahendragarh & 55.56 & 100.00 \\
\hline & & & Rewari & 43.68 & 84.35 \\
\hline & \multirow{2}{*}{ Rohtak } & \multirow{2}{*}{$1,341,953$} & Rewari & 5.79 & 15.65 \\
\hline & & & Rohtak & 94.21 & 82.44 \\
\hline & Sirsa & 707,068 & Sirsa & 100.00 & 100.00 \\
\hline & \multirow{2}{*}{ Sonipat } & \multirow{2}{*}{846,765} & Rohtak & 30.96 & 17.10 \\
\hline & & & Sonipat & 69.04 & 100.00 \\
\hline \multirow{6}{*}{ Himachal Pradesh } & Chamba & 311,147 & Chamba & 100.00 & 100.00 \\
\hline & Lahul And Spiti & 32,100 & Lahul And Spiti & 100.00 & 100.00 \\
\hline & Shimla & 510,932 & Shimla & 100.00 & 100.00 \\
\hline & \multirow{2}{*}{ Sirmaur } & 306952 & Sirmaur & 99.98 & 100.00 \\
\hline & & 306,952 & Solan & 0.02 & 0.02 \\
\hline & Solan & 303,280 & Solan & 100.00 & 99.98 \\
\hline & Badgam & 367,262 & Badgam & 100.00 & 100.00 \\
\hline & Baramula (Kashmir North) & 670,142 & Baramula & 100.00 & 100.00 \\
\hline & Jammu & 943,395 & Jammu & 100.00 & 100.00 \\
\hline Jammu \& Kashmir & Kathua & 369,123 & Kathua & 100.00 & 100.00 \\
\hline & Kupwara & 328,743 & Kupwara & 100.00 & 100.00 \\
\hline & Srinagar & 708,328 & Srinagar & 100.00 & 100.00 \\
\hline & Udhampur & 453,636 & Udhampur & 100.00 & 100.00 \\
\hline & Belgaum & 2.980 .440 & Belgaum & 99.95 & 100.00 \\
\hline & Deigaumi & $2,900,440$ & Uttar Kannad & 0.05 & 0.14 \\
\hline Karnataka & Bellary & $1,489,225$ & Bellary & 100.00 & 100.00 \\
\hline & Chitradurga & $1,777,499$ & Chitradurga & 100.00 & 100.00 \\
\hline & Shimoga & $1,656,731$ & Shimoga & 100.00 & 100.00 \\
\hline & Uttar Kannad & $1,072,034$ & Uttar Kannad & 100.00 & 99.86 \\
\hline
\end{tabular}


Table 6b. Boundary Changes in "Other" Districts: 1981-1991

\begin{tabular}{|c|c|c|c|c|c|}
\hline $\begin{array}{c}\text { Census } 1981 \\
\text { State/UT }\end{array}$ & 1981 District & $\begin{array}{c}\text { Population in } \\
1981\end{array}$ & 1991 District & $\begin{array}{c}\text { Share of } \\
1991 \\
\text { District in } \\
1981 \text { District }\end{array}$ & \begin{tabular}{|c} 
Share of \\
1981 District \\
in 1991 \\
District
\end{tabular} \\
\hline [1] & [2] & [3] & {$[4]$} & [5] & [6] \\
\hline \multirow{17}{*}{ Kerala } & \multirow{3}{*}{ Alleppey } & \multirow{3}{*}{$2,350,145$} & Alappuzha & 79.38 & 100.00 \\
\hline & & & Kollam & 0.01 & 0.01 \\
\hline & & & Pathanamthitta & 20.62 & 43.06 \\
\hline & \multirow{2}{*}{ Cannanore } & \multirow{2}{*}{$2,803,467$} & Kannur & 68.87 & 100.00 \\
\hline & & & Kasaragod & 31.13 & 100.00 \\
\hline & \multirow{2}{*}{ Ernakulam } & \multirow{2}{*}{$2,535,294$} & Ernakulam & 99.996 & 100.00 \\
\hline & & & Thrissur & 0.004 & 0.004 \\
\hline & \multirow{2}{*}{ Idukki } & \multirow{2}{*}{971,636} & Idukki & 99.76 & 100.00 \\
\hline & & & Pathanamthitta & 0.24 & 0.21 \\
\hline & Kottayam & $1,697,442$ & Kottayam & 100.00 & 100.00 \\
\hline & Kozhikode & $2,245,265$ & Kozhikode & 100.00 & 100.00 \\
\hline & Malappuram & $2,402,701$ & Malappuram & 100.00 & 100.00 \\
\hline & Palghat & $2,044,399$ & Palakkad & 100.00 & 100.00 \\
\hline & \multirow{2}{*}{ Quilon } & \multirow{2}{*}{$2,813,650$} & Kollam & 77.31 & 99.99 \\
\hline & & & Pathanamthitta & 22.69 & 56.73 \\
\hline & Trichur & $2,439,543$ & Thrissur & 100.00 & 99.996 \\
\hline & Wayanad & 554,026 & Wayanad & 100.00 & 100.00 \\
\hline \multirow{8}{*}{ Madhya Pradesh } & Bhopal & 894,739 & Bhopal & 100.00 & 100.00 \\
\hline & Bilaspur & $2,953,366$ & Bilaspur & 100.00 & 100.00 \\
\hline & Datia & 311,893 & Datia & 100.00 & 100.00 \\
\hline & Durg & $1,890,467$ & Durg & 100.00 & 100.00 \\
\hline & Gwalior & $1,107,879$ & Gwalior & 100.00 & 100.00 \\
\hline & Hoshangabad & $1,003,939$ & Hoshangabad & 100.00 & 100.00 \\
\hline & Raj Nandgaon & $1,167,501$ & Rajnandgaon & 100.00 & 100.00 \\
\hline & Sehore & 657,381 & Sehore & 100.00 & 100.00 \\
\hline \multirow{21}{*}{ Maharashtra } & Ahmadnagar & $2,708,309$ & Ahmednagar & 100.00 & 99.85 \\
\hline & \multirow{3}{*}{ Aurangabad } & \multirow{3}{*}{$2,433,420$} & Ahmednagar & 0.17 & 0.15 \\
\hline & & & Aurangabad & 65.17 & 100.00 \\
\hline & & & Jalna & 34.66 & 81.87 \\
\hline & \multirow{2}{*}{ Bid } & \multirow{2}{*}{$1,486,030$} & Bid & 95.08 & 100.00 \\
\hline & & & Latur & 4.92 & 5.65 \\
\hline & \multirow{3}{*}{ Chandrapur } & \multirow{3}{*}{$2,055,642$} & Adilabad & 0.07 & 0.08 \\
\hline & & & Chandrapur & 68.93 & 100.00 \\
\hline & & & Gadchiroli & 31.00 & 100.00 \\
\hline & \multirow{2}{*}{ Kolhapur } & \multirow{2}{*}{$2,506,330$} & Kolhapur & 98.04 & 100.00 \\
\hline & & & Sindhudurg & 1.96 & 6.30 \\
\hline & \multirow{2}{*}{ Osmanabad } & 2230620 & Latur & 54.69 & 94.35 \\
\hline & & $2,230,620$ & Osmanabad & 45.31 & 98.16 \\
\hline & Parbhani & $1,829,378$ & Jalna & 10.21 & 18.13 \\
\hline & |r aminamin & $1,8<9,3 / 8$ & Parbhani & 89.79 & 100.00 \\
\hline & Ratnaqiri & 2.111 .311 & Ratnagiri & 65.35 & 100.00 \\
\hline & nalmagin & $2,111,311$ & Sindhudurg & 34.65 & 93.70 \\
\hline & Sangli & $1,831,212$ & Sangli & 100.00 & 99.83 \\
\hline & & & Osmanabad & 0.73 & 1.84 \\
\hline & Solapur & $2,610,144$ & Sangli & 0.12 & 0.17 \\
\hline & & & Solapur & 99.16 & 100.00 \\
\hline
\end{tabular}


Table 6b. Boundary Changes in "Other" Districts: 1981-1991

\begin{tabular}{|c|c|c|c|c|c|}
\hline $\begin{array}{c}\text { Census } 1981 \\
\text { State/UT }\end{array}$ & 1981 District & $\begin{array}{c}\text { Population in } \\
1981\end{array}$ & 1991 District & $\begin{array}{c}\text { Share of } \\
1991 \\
\text { District in } \\
1981 \text { District }\end{array}$ & $\begin{array}{c}\text { Share of } \\
1981 \text { District } \\
\text { in } 1991 \\
\text { District }\end{array}$ \\
\hline [1] & [2] & [3] & [4] & [5] & {$[6]$} \\
\hline \multirow{11}{*}{ Manipur } & \multirow{3}{*}{ Manipur Central } & \multirow{3}{*}{929,077} & Bishnupur & 15.19 & 99.57 \\
\hline & & & Imphal & 59.86 & 99.80 \\
\hline & & & Thoubal & 24.95 & 99.62 \\
\hline & \multirow{4}{*}{ Manipur North } & \multirow{4}{*}{155,421} & Bishnupur & 0.21 & 0.23 \\
\hline & & & Imphal & 0.71 & 0.20 \\
\hline & & & Senapati & 98.50 & 100.00 \\
\hline & & & Thoubal & 0.58 & 0.38 \\
\hline & \multirow{2}{*}{ Manipur South } & \multirow{2}{*}{134,776} & Bishnupur & 0.21 & 0.20 \\
\hline & & & Churachandpur & 99.79 & 100.00 \\
\hline & Manipur West & 62,289 & Tamenglong & 100.00 & 100.00 \\
\hline & Tengnoupal & 56,444 & Chandel & 100.00 & 100.00 \\
\hline \multirow{3}{*}{ Meghalaya } & East Khasi Hills & 511,414 & East Khasi Hills & 100.00 & 100.00 \\
\hline & Jaintia Hills & 156,402 & Jaintia Hills & 100.00 & 100.00 \\
\hline & West Khasi Hills & 161,576 & West Khasi Hills & 100.00 & 100.00 \\
\hline \multirow{10}{*}{ Nagaland } & \multirow{2}{*}{ Kohima } & \multirow{2}{*}{250,105} & Kohima & 95.46 & 100.00 \\
\hline & & & Zunheboto & 4.54 & 15.66 \\
\hline & \multirow{2}{*}{ Mokokchung } & \multirow{2}{*}{104,193} & Mokokchung & 99.74 & 100.00 \\
\hline & & & Wokha & 0.26 & 0.46 \\
\hline & Mon & 78,938 & Mon & 100.00 & 83.83 \\
\hline & Phek & 70,618 & Phek & 100.00 & 100.00 \\
\hline & \multirow{2}{*}{ Tuensang } & \multirow{2}{*}{152,332} & Mon & 9.99 & 16.17 \\
\hline & & & Tuensang & 90.01 & 100.00 \\
\hline & Wokha & 57,583 & Wokha & 100.00 & 99.54 \\
\hline & Zunheboto & 61,161 & Zunheboto & 100.00 & 84.34 \\
\hline \multirow{14}{*}{ Punjab } & Amritsar & $2,118,490$ & Amritsar & 100.00 & 100.00 \\
\hline & Bathinda & $1,304,606$ & Bathinda & 100.00 & 100.00 \\
\hline & Faridkot & $1,436,228$ & Faridkot & 100.00 & 99.79 \\
\hline & \multirow{2}{*}{ Firozpur } & \multirow{2}{*}{$1,307,804$} & Faridkot & 0.24 & 0.21 \\
\hline & & & Firozpur & 99.76 & 100.00 \\
\hline & Gurdaspur & $1,513,435$ & Gurdaspur & 100.00 & 100.00 \\
\hline & Hoshiarpur & $1,243,807$ & Hoshiarpur & 100.00 & 99.87 \\
\hline & Jalandhar & $1,734,574$ & Jalandhar & 100.00 & 100.00 \\
\hline & Ludhiana & 1.818 .912 & Hoshiarpur & 0.07 & 0.10 \\
\hline & Luniria & מוט,סוס,ו & Ludhiana & 99.93 & 100.00 \\
\hline & Patiala & $1,568,898$ & Patiala & 100.00 & 100.00 \\
\hline & Runnagar & 716662 & Hoshiarpur & 0.04 & 0.02 \\
\hline & Rupriagar & 110,002 & Rupnagar & 99.96 & 100.00 \\
\hline & Sangrur & $1,410,250$ & Sangrur & 100.00 & 100.00 \\
\hline & Alwar & $1,771,173$ & Alwar & 99.12 & 100.00 \\
\hline & Alvval & טדוסודות, & Jaipur & 0.88 & 0.45 \\
\hline & Bundi & 586,982 & Bundi & 100.00 & 95.91 \\
\hline & Chittaurgarh & $1,232,494$ & Chittorgarh & 100.00 & 100.00 \\
\hline & Jaipur & $3,420,574$ & Jaipur & 100.00 & 99.55 \\
\hline Raiasthan & Jaisalmer & 243,082 & Jaisalmer & 100.00 & 100.00 \\
\hline najastnan & Jhalawar & 784,998 & Jhalawar & 100.00 & 100.00 \\
\hline & Jodhpur & $1,667,791$ & Jodhpur & 100.00 & 100.00 \\
\hline & Kota & $1.559,784$ & Bundi & 1.61 & 4.09 \\
\hline & ruta & $1,000,104$ & Kota & 98.39 & 100.00 \\
\hline & Sawai Madhopur & $1,535,870$ & Sawai Madhopur & 100.00 & 100.00 \\
\hline & Udaipur & $2,356,959$ & Udaipur & 100.00 & 100.00 \\
\hline
\end{tabular}


Table 6b. Boundary Changes in "Other" Districts: 1981-1991

\begin{tabular}{|c|c|c|c|c|c|}
\hline $\begin{array}{c}\text { Census } 1981 \\
\text { State/UT }\end{array}$ & 1981 District & $\begin{array}{c}\text { Population in } \\
1981\end{array}$ & 1991 District & $\begin{array}{c}\text { Share of } \\
1991 \\
\text { District in } \\
1981 \text { District }\end{array}$ & $\begin{array}{c}\text { Share of } \\
1981 \text { District } \\
\text { in } 1991 \\
\text { District }\end{array}$ \\
\hline [1] & {$[2]$} & {$[3]$} & [4] & [5] & [6] \\
\hline \multirow{2}{*}{ Sikkim } & South District & 75,976 & South District & 100.00 & 100.00 \\
\hline & West District & 75,192 & West District & 100.00 & 100.00 \\
\hline \multirow{8}{*}{ Tamil Nadu } & Chengalpattu & $3,616,508$ & Chengalpattu-Mgr & 100.00 & 100.00 \\
\hline & Madras & $3,276,622$ & Madras & 100.00 & 100.00 \\
\hline & Pudukkottai & $1,156,813$ & Puddukkottai & 100.00 & 100.00 \\
\hline & \multirow{3}{*}{ Ramanathapuram } & \multirow{3}{*}{$3,335,437$} & Kamarajar & 40.20 & 100.00 \\
\hline & & & Pasumpon M. Thevar & 29.15 & 100.00 \\
\hline & & & Ramanathapuram & 30.65 & 100.00 \\
\hline & Thanjavur & $4,063,545$ & Thanjavur & 100.00 & 100.00 \\
\hline & Tiruchchirappallic & $3,612,320$ & Tiruchirapalli & 100.00 & 100.00 \\
\hline \multirow{4}{*}{ Tripura } & \multirow{2}{*}{ North Tripura } & \multirow{2}{*}{541,248} & North Tripura & 98.51 & 100.00 \\
\hline & & & South Tripura & 1.49 & 1.48 \\
\hline & South Tripura & 535,558 & South Tripura & 100.00 & 98.52 \\
\hline & West Tripura & 976,252 & West Tripura & 100.00 & 100.00 \\
\hline \multirow{36}{*}{ Uttar Pradesh } & \multirow{2}{*}{ Agra } & \multirow{2}{*}{$2,852,942$} & Agra & 79.11 & 100.00 \\
\hline & & & Firozabad & 20.89 & 47.29 \\
\hline & Aligarh & $2,574,925$ & Aligarh & 100.00 & 100.00 \\
\hline & Almora & 757,373 & Almora & 100.00 & 100.00 \\
\hline & \multirow{2}{*}{ Azamgarh } & \multirow{2}{*}{$3,544,130$} & Azamgarh & 70.90 & 100.00 \\
\hline & & & Mau & 29.10 & 91.56 \\
\hline & \multirow{2}{*}{ Ballia } & \multirow{2}{*}{$1,945,376$} & Ballia & 95.11 & 100.00 \\
\hline & & & Mau & 4.89 & 8.44 \\
\hline & Bara Banki & $1,992,074$ & Barabanki & 100.00 & 100.00 \\
\hline & \multirow{2}{*}{ Basti } & \multirow{2}{*}{$3,578,069$} & Basti & 61.43 & 100.00 \\
\hline & & & Siddharth Nagar & 38.57 & 100.00 \\
\hline & \multirow{2}{*}{ Bijnor } & \multirow{2}{*}{$1,939,261$} & Bijnor & 99.37 & 100.00 \\
\hline & & & Hardwar & 0.63 & 1.37 \\
\hline & Bulandshahr & $2,358,270$ & Bulandshahr & 100.00 & 99.99 \\
\hline & Chamoli & 364,346 & Chamoli & 100.00 & 97.52 \\
\hline & Etawah & $1,742,651$ & Etawah & 100.00 & 100.00 \\
\hline & Faizabad & $2,382,515$ & Faizabad & 100.00 & 100.00 \\
\hline & Fatehpur & $1,572,421$ & Fatehpur & 100.00 & 100.00 \\
\hline & \multirow{2}{*}{ Garhwal } & \multirow{2}{*}{637,877} & Chamoli & 1.45 & 2.48 \\
\hline & & & Garhwal & 98.55 & 100.00 \\
\hline & Ghaziabad & $1,843,130$ & Ghaziabad & 100.00 & 99.99 \\
\hline & & 3742223 & Kanpur Dehat & 47.87 & 100.00 \\
\hline & Rampur & $3,14<, 2<3$ & Kanpur Nagar & 52.13 & 100.00 \\
\hline & Mainpuri & $1,726,202$ & Firozabad & 38.48 & 52.71 \\
\hline & jivampunt & $1,1<0,20<$ & Mainpuri & 61.52 & 100.00 \\
\hline & Mathura & $1,560,447$ & Mathura & 100.00 & 100.00 \\
\hline & Meerut & $2,767,246$ & Meerut & 100.00 & 100.00 \\
\hline & Muzaffarnagar & 2.274 .487 & Hardwar & 1.14 & 2.92 \\
\hline & IVicuzanamiagai & $2,214,401$ & Muzaffarnagar & 98.86 & 100.00 \\
\hline & Naini Tal & $1,136,523$ & Nainital & 100.00 & 100.00 \\
\hline & Pithoragarh & 489,267 & Pithoragarh & 100.00 & 100.00 \\
\hline & Rae Bareli & $1,886,940$ & Rae Bareli & 100.00 & 100.00 \\
\hline & Saharanpur & $2,673,561$ & Hardwar & 31.87 & 95.70 \\
\hline & (1) & $2,010,001$ & Saharanpur & 68.13 & 100.00 \\
\hline & Sultanpur & $2,042,778$ & Sultanpur & 100.00 & 100.00 \\
\hline & Tehri Garhwal & 497,710 & Tehri Garhwal & 100.00 & 100.00 \\
\hline
\end{tabular}


Table 6b. Boundary Changes in "Other" Districts: 1981-1991

\begin{tabular}{|c|c|c|c|c|c|}
\hline $\begin{array}{c}\text { Census } 1981 \\
\text { State/UT }\end{array}$ & 1981 District & $\begin{array}{c}\text { Population in } \\
1981\end{array}$ & 1991 District & \begin{tabular}{|c} 
Share of \\
1991 \\
District in \\
1981 District
\end{tabular} & \begin{tabular}{|c} 
Share of \\
1981 District \\
in 1991 \\
District
\end{tabular} \\
\hline [1] & [2] & {$[3]$} & [4] & {$[5]$} & [6] \\
\hline \multirow{6}{*}{ West Bengal } & Calcutta & $3,305,006$ & Calcutta & 100.00 & 80.09 \\
\hline & Murshidabad & $3,697,552$ & Murshidabad & 100.00 & 100.00 \\
\hline & Nadia & $2,964,253$ & Nadia & 100.00 & 100.00 \\
\hline & \multirow{3}{*}{ Twenty Four Parganas } & \multirow{3}{*}{$10,739,439$} & Calcutta & 7.65 & 19.91 \\
\hline & & & North 24 Paraganas & 51.49 & 100.00 \\
\hline & & & South 24 Paraganas & 40.86 & 100.00 \\
\hline
\end{tabular}

a portions of two villages, with unknown population and a total area of $0.73 \mathrm{sq}$. $\mathrm{km}$., were transferred from Gandhinagar district to Ahmadabad district between 1981 and 1991

${ }^{\mathrm{b}}$ Our work relies on the Haryana population transfers listed in Statement 1 (p. 118) and the Appendix to Table A-2 published in Government of India (1996). These figures do not include some small transfers that are listed in Appendix 1 to Table A-1. For this reason the weights we have listed for Haryana districts (except Sirsa and Bhiwani) are

${ }^{c}$ an uninhabited portion of one village, with an area of 0.04 sq. $\mathrm{km}$., was transferred from Tirucchirappalli district to Pasumpon M. Thevar district between 1981 and 1991. 
Table 6c. Boundary Changes in "Other" Districts: 1991-2001

\begin{tabular}{|c|c|c|c|c|c|}
\hline Census 1991 State/UT & 1991 District & $\begin{array}{c}\text { Population in } \\
1991\end{array}$ & 2001 District & $\begin{array}{c}\text { Share of } \\
2001 \\
\text { District in } \\
1991 \\
\text { District }\end{array}$ & $\begin{array}{c}\text { Share of } \\
1991 \\
\text { District in } \\
2001 \\
\text { District }\end{array}$ \\
\hline [1] & [2] & [3] & [4] & [5] & [6] \\
\hline \multirow{11}{*}{ Andhra Pradesh } & Adilabad & $2,082,479$ & Adilabad & 100.00 & 100.00 \\
\hline & \multirow{2}{*}{ Anantapur } & \multirow{2}{*}{$3,183,814$} & Anantapur & 99.91 & 100.00 \\
\hline & & & Cuddapah & 0.09 & 0.13 \\
\hline & Chittoor & $3,261,118$ & Chittoor & 100.00 & 100.00 \\
\hline & Cuddapah & $2,267,769$ & Cuddapah & 100.00 & 99.87 \\
\hline & Hyderabad & $3,145,939$ & Hyderabad & 100.00 & 100.00 \\
\hline & Nellore & $2,392,260$ & Nellore & 100.00 & 100.00 \\
\hline & Rangareddi & $2,551,966$ & Rangareddi & 100.00 & 100.00 \\
\hline & Srikakulam & $2,321,126$ & Srikakulam & 100.00 & 100.00 \\
\hline & Visakhapatanam & $3,285,092$ & Visakhapatnam & 100.00 & 100.00 \\
\hline & Vizianagaram & $2,110,943$ & Vizianagaram & 100.00 & 100.00 \\
\hline \multirow{6}{*}{ Arunachal Pradesh } & \multirow{2}{*}{ East Siang } & \multirow{2}{*}{99,643} & East Siang & 72.12 & 100.00 \\
\hline & & & Upper Siang & 27.88 & 100.00 \\
\hline & \multirow{2}{*}{ Lower Subansiri } & \multirow{2}{*}{155,978} & Lower Subansiri & 53.32 & 100.00 \\
\hline & & & Papum Pare & 46.68 & 100.00 \\
\hline & Upper Subansiri & 50,086 & Upper Subansiri & 100.00 & 100.00 \\
\hline & West Siang & 89,936 & West Siang & 100.00 & 100.00 \\
\hline \multirow{15}{*}{ Assam } & Barpeta & $1,385,659$ & Barpeta & 100.00 & 100.00 \\
\hline & Bongaigaon & 807,523 & Bongaigaon & 100.00 & 100.00 \\
\hline & Darrang & $1,298,860$ & Darrang & 100.00 & 100.00 \\
\hline & \multirow{2}{*}{ Dhubri } & \multirow{2}{*}{$1,332,475$} & Dhubri & 99.39 & 100.00 \\
\hline & & & Kokrajhar & 0.61 & 1.00 \\
\hline & Goalpara & 668,138 & Goalpara & 100.00 & 100.00 \\
\hline & Golaghat & 828,096 & Golaghat & 100.00 & 100.00 \\
\hline & Jorhat & 871,206 & Jorhat & 100.00 & 100.00 \\
\hline & Kamrup & $2,000,071$ & Kamrup & 100.00 & 100.00 \\
\hline & Kokrajhar & 800,659 & Kokrajhar & 100.00 & 99.00 \\
\hline & Marigaon & 639,682 & Marigaon & 100.00 & 100.00 \\
\hline & Nagaon & $1,893,171$ & Nagaon & 100.00 & 100.00 \\
\hline & Nalbari & $1,016,390$ & Nalbari & 100.00 & 100.00 \\
\hline & Sibsagar & 907,983 & Sibsagar & 100.00 & 100.00 \\
\hline & Sonitpur & $1,424,287$ & Sonitpur & 100.00 & 100.00 \\
\hline \multirow{16}{*}{ Bihar } & Begusarai & $1,814,773$ & Begusarai & 100.00 & 100.00 \\
\hline & \multirow{2}{*}{ Dhanbad } & \multirow{2}{*}{$2,674,651$} & Bokaro & 27.11 & 49.86 \\
\hline & & & Dhanbad & 72.89 & 100.00 \\
\hline & \multirow{2}{*}{ Giridih } & \multirow{2}{*}{$2,225,480$} & Bokaro & 32.77 & 50.14 \\
\hline & & & Giridih & 67.23 & 100.00 \\
\hline & \multirow{3}{*}{ Hazaribag } & \multirow{3}{*}{$2,843,544$} & Chatra & 21.55 & 100.00 \\
\hline & & & Hazaribagh & 64.57 & 100.00 \\
\hline & & & Kodarma & 13.88 & 100.00 \\
\hline & Khagaria & 987,227 & Khagaria & 100.00 & 100.00 \\
\hline & \multirow{4}{*}{ Munger } & \multirow{4}{*}{$3,060,027$} & Jamui & 34.36 & 100.00 \\
\hline & & & Lakhisarai & 21.12 & 100.00 \\
\hline & & & Munger & 30.84 & 100.00 \\
\hline & & & Sheikhpura & 13.68 & 99.59 \\
\hline & \multirow{2}{*}{ Nalanda } & 1997995 & Nalanda & 99.91 & 100.00 \\
\hline & & $1,991,995$ & Sheikhpura & 0.09 & 0.41 \\
\hline & Patna & $3,618,211$ & Patna & 100.00 & 100.00 \\
\hline
\end{tabular}


Table 6c. Boundary Changes in "Other" Districts: 1991-2001

\begin{tabular}{|c|c|c|c|c|c|}
\hline Census 1991 State/UT & 1991 District & $\begin{array}{c}\text { Population in } \\
1991\end{array}$ & 2001 District & $\begin{array}{c}\text { Share of } \\
2001 \\
\text { District in } \\
1991 \\
\text { District }\end{array}$ & $\begin{array}{c}\text { Share of } \\
1991 \\
\text { District in } \\
2001 \\
\text { District }\end{array}$ \\
\hline [1] & {$[2]$} & {$[3]$} & [4] & [5] & [6] \\
\hline \multirow{21}{*}{ Gujarat } & \multirow{2}{*}{ Ahmadabad } & \multirow{2}{*}{$4,801,812$} & Ahmadabad & 95.54 & 100.00 \\
\hline & & & Gandhinagar & 4.46 & 19.89 \\
\hline & \multirow{2}{*}{ Amreli } & \multirow{2}{*}{$1,252,589$} & Amreli & 86.76 & 83.03 \\
\hline & & & Junagadh & 13.24 & 7.93 \\
\hline & \multirow{2}{*}{ Banas Kantha } & \multirow{2}{*}{$2,162,578$} & Banas Kantha & 91.63 & 100.00 \\
\hline & & & Patan & 8.37 & 17.48 \\
\hline & \multirow{2}{*}{ Bharuch } & \multirow{2}{*}{$1,546,145$} & Bharuch & 74.27 & 100.00 \\
\hline & & & Narmada & 25.73 & 88.54 \\
\hline & \multirow{2}{*}{ Bhavnagar } & \multirow{2}{*}{$2,292,026$} & Amreli & 9.69 & 16.97 \\
\hline & & & Bhavnagar & 90.31 & 100.00 \\
\hline & Gandhinagar & 408,992 & Gandhinagar & 100.00 & 37.96 \\
\hline & \multirow{2}{*}{ Junagadh } & \multirow{2}{*}{$2,394,859$} & Junagadh & 80.40 & 92.07 \\
\hline & & & Porbandar & 19.60 & 100.00 \\
\hline & \multirow{3}{*}{ Kheda } & \multirow{3}{*}{$3,440,897$} & Anand & 47.74 & 100.00 \\
\hline & & & Gandhinagar & 0.33 & 1.07 \\
\hline & & & Kheda & 51.93 & 100.00 \\
\hline & \multirow{3}{*}{ Mahasana } & \multirow{3}{*}{$2,937,810$} & Gandhinagar & 15.07 & 41.08 \\
\hline & & & Mahesana & 55.83 & 100.00 \\
\hline & & & Patan & 29.10 & 82.52 \\
\hline & Vadodara & 3089610 & Narmada & 1.67 & 11.46 \\
\hline & vadodara & & Vadodara & 98.33 & 100.00 \\
\hline & Ambala & 1116878 & Ambala & 72.21 & 100.00 \\
\hline & Ambala & $1,116,8 / 8$ & Panchkula & 27.79 & 100.00 \\
\hline & Bhiwani & 1139718 & Bhiwani & 99.81 & 97.78 \\
\hline & Bniwanı & $1,139,118$ & Hisar & 0.19 & 0.18 \\
\hline & Faridabad & $1,477,240$ & Faridabad & 100.00 & 100.00 \\
\hline & Gurgaon & $1,146,090$ & Gurgaon & 100.00 & 100.00 \\
\hline & Hicar & 1844634 & Fatehabad & 35.03 & 100.00 \\
\hline & & 1,844,634 & Hisar & 64.97 & 99.11 \\
\hline & & & Jind & 98.08 & 96.35 \\
\hline & Jind & 963,104 & Kaithal & 1.05 & 1.29 \\
\hline & & & Karnal & 0.87 & 0.81 \\
\hline & & & Jind & 3.82 & 3.20 \\
\hline & & & Kaithal & 93.56 & 98.21 \\
\hline & Kaithal & 820,685 & Karnal & 0.96 & 0.76 \\
\hline & & & Kurukshetra & 1.66 & 2.03 \\
\hline & & & Karnal & 97.85 & 83.71 \\
\hline & Karnal & 885,797 & Kurukshetra & 0.24 & 0.31 \\
\hline Haryana & & & Panipat & 1.91 & 2.43 \\
\hline & Kurukshetra & 641943 & Kaithal & 0.61 & 0.50 \\
\hline & & & Kurukshetra & 99.39 & 95.32 \\
\hline & Mahendragarh & 681,869 & Mahendragarh & 100.00 & 100.00 \\
\hline & & & Karnal & 18.28 & 14.71 \\
\hline & Panipat & 833,501 & Panipat & 81.72 & 97.57 \\
\hline & & & Jhajjar & 2.04 & 1.77 \\
\hline & Rewari & 623,301 & Rewari & 97.96 & 100.00 \\
\hline & & & Bhiwani & 1.43 & 2.22 \\
\hline & & & Hisar & 0.47 & 0.71 \\
\hline & & 1000606 & Jhajjar & 38.84 & 98.23 \\
\hline & Rohtak & $1,808,606$ & Jind & 0.25 & 0.45 \\
\hline & & & Rohtak & 42.96 & 100.00 \\
\hline & & & Sonipat & 16.05 & 27.77 \\
\hline & Sirsa & 903,536 & Sirsa & 100.00 & 100.00 \\
\hline & Sonipat & 754,866 & Sonipat & 100.00 & 72.23 \\
\hline & Yamunanogr & 021000 & Kurukshetra & 1.90 & 2.33 \\
\hline & Yamunanagar & 821,880 & Yamunanagar & 98.10 & 100.00 \\
\hline
\end{tabular}


Table 6c. Boundary Changes in "Other" Districts: 1991-2001

\begin{tabular}{|c|c|c|c|c|c|}
\hline Census 1991 State/UT & 1991 District & $\begin{array}{c}\text { Population in } \\
1991\end{array}$ & 2001 District & $\begin{array}{c}\text { Share of } \\
2001 \\
\text { District in } \\
1991 \\
\text { District }\end{array}$ & $\begin{array}{c}\text { Share of } \\
1991 \\
\text { District in } \\
2001 \\
\text { District }\end{array}$ \\
\hline$[1]$ & [2] & [3] & [4] & [5] & {$[6]$} \\
\hline \multirow{5}{*}{ Himachal Pradesh } & Chamba & 393,286 & Chamba & 100.00 & 100.00 \\
\hline & Lahul And Spiti & 31,294 & Lahul \& Spiti & 100.00 & 100.00 \\
\hline & Shimla & 617,404 & Shimla & 100.00 & 100.00 \\
\hline & Sirmaur & 379,695 & Sirmaur & 100.00 & 100.00 \\
\hline & Solan & 382,268 & Solan & 100.00 & 100.00 \\
\hline \multirow{7}{*}{ Jammu \& Kashmir* } & Badgam & 483,698 & Badgam & 100.00 & 100.00 \\
\hline & Baramula & 891,044 & Baramula & 100.00 & 100.00 \\
\hline & Jammu & $1,231,553$ & Jammu & 100.00 & 100.00 \\
\hline & Kathua & 452,739 & Kathua & 100.00 & 100.00 \\
\hline & Kupwara & 465,948 & Kupwara & 100.00 & 100.00 \\
\hline & Srinagar & 928,526 & Srinagar & 100.00 & 100.00 \\
\hline & Udhampur & 584,051 & Udhampur & 100.00 & 100.00 \\
\hline \multirow{8}{*}{ Karnataka } & Belgaum & $3,583,606$ & Belgaum & 100.00 & 100.00 \\
\hline & \multirow{2}{*}{ Bellary } & \multirow{2}{*}{$1,890,092$} & Bellary & 87.61 & 100.00 \\
\hline & & & Davanagere & 12.39 & 15.01 \\
\hline & \multirow{2}{*}{ Chitradurga } & \multirow{2}{*}{$2,180,443$} & Chitradurga & 60.20 & 100.00 \\
\hline & & & Davanagere & 39.80 & 55.65 \\
\hline & \multirow{2}{*}{ Shimoga } & \multirow{2}{*}{$1,909,663$} & Davanagere & 23.95 & 29.34 \\
\hline & & & Shimoga & 76.05 & 100.00 \\
\hline & Uttar Kannad & $1,220,260$ & Uttara Kannada & 100.00 & 100.00 \\
\hline \multirow{14}{*}{ Kerala } & Alappuzha $^{\mathrm{a}}$ & $2,001,217$ & Alappuzha & 100.00 & 100.00 \\
\hline & Ernakulam $^{\mathrm{b}}$ & $2,817,236$ & Ernakulam & 100.00 & 99.19 \\
\hline & \multirow{2}{*}{ Idukki } & \multirow{2}{*}{$1,078,066$} & Ernakulam & 2.14 & 0.81 \\
\hline & & & Idukki & 97.86 & 100.00 \\
\hline & Kannur & $2,251,727$ & Kannur & 100.00 & 100.00 \\
\hline & Kasaragod & $1,071,508$ & Kasaragod & 100.00 & 100.00 \\
\hline & Kollam $^{\mathrm{C}}$ & $2,407,566$ & Kollam & 100.00 & 100.00 \\
\hline & Kottayam & $1,828,271$ & Kottayam & 100.00 & 100.00 \\
\hline & Kozhikode & $2,619,941$ & Kozhikode & 100.00 & 100.00 \\
\hline & Malappuram & $3,096,330$ & Malappuram & 100.00 & 100.00 \\
\hline & Palakkad & $2,382,235$ & Palakkad & 100.00 & 100.00 \\
\hline & Pathanamthitta $^{d}$ & $1,188,332$ & Pathanamthitta & 100.00 & 100.00 \\
\hline & Thrissur & $2,737,311$ & Thrissur & 100.00 & 100.00 \\
\hline & Wayanad & 672,128 & Wayanad & 100.00 & 100.00 \\
\hline \multirow{14}{*}{ Madhya Pradesh } & Bhopal & $1,351,479$ & Bhopal & 100.00 & 100.00 \\
\hline & \multirow{4}{*}{ Bilaspur } & \multirow{4}{*}{$3,793,566$} & Bilaspur & 44.68 & 100.00 \\
\hline & & & Janjgir - Champa & 29.27 & 100.00 \\
\hline & & & Kawardha & 4.29 & 31.66 \\
\hline & & & Korba & 21.77 & 100.00 \\
\hline & Datia & 396,317 & Datia & 100.00 & 76.90 \\
\hline & Durg & $2,397,134$ & Durg & 100.00 & 100.00 \\
\hline & \multirow{2}{*}{ Gwalior } & \multirow{2}{*}{$1,412,610$} & Datia & 8.43 & 23.10 \\
\hline & & & Gwalior & 91.57 & 100.00 \\
\hline & \multirow{2}{*}{ Hoshangabad } & \multirow{2}{*}{$1,267,211$} & Harda & 30.05 & 100.00 \\
\hline & & & Hoshangabad & 69.95 & 100.00 \\
\hline & \multirow{2}{*}{ Rajnandgaon } & \multirow{2}{*}{$1,439,951$} & Kawardha & 24.37 & 68.34 \\
\hline & & & Rajnandgaon & 75.63 & 100.00 \\
\hline & Sehore & 841,358 & Sehore & 100.00 & 100.00 \\
\hline
\end{tabular}


Table 6c. Boundary Changes in "Other" Districts: 1991-2001

\begin{tabular}{|c|c|c|c|c|c|}
\hline Census 1991 State/UT & 1991 District & $\begin{array}{c}\text { Population in } \\
1991\end{array}$ & 2001 District & $\begin{array}{c}\text { Share of } \\
2001 \\
\text { District in } \\
1991 \\
\text { District }\end{array}$ & $\begin{array}{c}\text { Share of } \\
1991 \\
\text { District in } \\
2001 \\
\text { District }\end{array}$ \\
\hline [1] & {$[2]$} & [3] & [4] & [5] & {$[6]$} \\
\hline \multirow{15}{*}{ Maharashtra } & Ahmednagar & $3,372,935$ & Ahmadnagar & 100.00 & 100.00 \\
\hline & Aurangabad & $2,213,779$ & Aurangabad & 100.00 & 100.00 \\
\hline & Bid & $1,822,072$ & Bid & 100.00 & 100.00 \\
\hline & Chandrapur & $1,771,994$ & Chandrapur & 100.00 & 100.00 \\
\hline & Gadchiroli & 787,010 & Gadchiroli & 100.00 & 100.00 \\
\hline & Jalna & $1,364,425$ & Jalna & 100.00 & 100.00 \\
\hline & Kolhapur & $2,989,507$ & Kolhapur & 100.00 & 100.00 \\
\hline & Latur & $1,676,641$ & Latur & 100.00 & 100.00 \\
\hline & Osmanabad & $1,276,327$ & Osmanabad & 100.00 & 100.00 \\
\hline & Parhhani & 2117035 & Hingoli & 38.92 & 100.00 \\
\hline & Parbnanı & $2,117,035$ & Parbhani & 61.08 & 100.00 \\
\hline & Ratnagiri & $1,544,057$ & Ratnagiri & 100.00 & 100.00 \\
\hline & Sangli & $2,209,488$ & Sangli & 100.00 & 100.00 \\
\hline & Sindhudurg & 832,152 & Sindhudurg & 100.00 & 100.00 \\
\hline & Solapur & $3,231,057$ & Solapur & 100.00 & 100.00 \\
\hline \multirow{8}{*}{ Manipur } & Bishnupur & 180,773 & Bishnupur & 100.00 & 100.00 \\
\hline & Chandel & 71,014 & Chandel & 100.00 & 100.00 \\
\hline & Churachandpur & 176,184 & Churachandpur & 100.00 & 100.00 \\
\hline & & & Imphal East & 46.46 & 100.00 \\
\hline & Imphal & 711,261 & Imphal West & 53.54 & 100.00 \\
\hline & Senapati & 208,406 & Senapati & 100.00 & 100.00 \\
\hline & Tamenglong & 86,278 & Tamenglong & 100.00 & 100.00 \\
\hline & Thoubal & 293,958 & Thoubal & 100.00 & 100.00 \\
\hline \multirow{5}{*}{ Meghalaya } & & & East Khasi Hills & 80.51 & 100.00 \\
\hline & East Khasi Hills & 665,218 & Ri Bhoi & 19.14 & 100.00 \\
\hline & & & West Khasi Hills & 0.35 & 1.06 \\
\hline & Jaintia Hills & 220,473 & Jaintia Hills & 100.00 & 100.00 \\
\hline & West Khasi Hills & 220,157 & West Khasi Hills & 100.00 & 98.94 \\
\hline \multirow{8}{*}{ Nagaland } & & & Dimapur & 45.91 & 100.00 \\
\hline & konima & 381,581 & Kohima & 54.09 & 100.00 \\
\hline & Mokokchung & 158,374 & Mokokchung & 100.00 & 100.00 \\
\hline & Mon & 149,699 & Mon & 100.00 & 100.00 \\
\hline & Phek & 102,156 & Phek & 100.00 & 100.00 \\
\hline & Tuensang & 232,906 & Tuensang & 100.00 & 100.00 \\
\hline & Wokha & 82,612 & Wokha & 100.00 & 100.00 \\
\hline & Zunheboto & 96,218 & Zunheboto & 100.00 & 100.00 \\
\hline \multirow{19}{*}{ Punjab } & Amritsar & $2,504,560$ & Amritsar & 100.00 & 99.98 \\
\hline & \multirow{2}{*}{ Bathinda } & \multirow{2}{*}{$1,559,963$} & Bathinda & 63.16 & 100.00 \\
\hline & & & Mansa & 36.84 & 100.00 \\
\hline & \multirow{3}{*}{ Faridkot } & \multirow{3}{*}{$1,730,876$} & Faridkot & 26.29 & 100.00 \\
\hline & & & Moga & 35.98 & 80.06 \\
\hline & & & Muktsar & 37.73 & 99.79 \\
\hline & \multirow{5}{*}{ Firozpur } & \multirow{5}{*}{$1,607,817$} & Amritsar & 0.03 & 0.02 \\
\hline & & & Firozpur & 90.12 & 100.00 \\
\hline & & & Jalandhar & 0.12 & 0.12 \\
\hline & & & Moga & 9.65 & 19.94 \\
\hline & & & Muktsar & 0.08 & 0.21 \\
\hline & Gurdaspur & $1,756,732$ & Gurdaspur & 100.00 & 99.97 \\
\hline & \multirow{4}{*}{ Hoshiarpur } & \multirow{4}{*}{$1,455,028$} & Gurdaspur & 0.04 & 0.03 \\
\hline & & & Hoshiarpur & 89.26 & 100.00 \\
\hline & & & Ludhiana & 0.14 & 0.08 \\
\hline & & & Nawanshahr & 10.57 & 28.94 \\
\hline & \multirow{3}{*}{ Jalandhar } & \multirow{3}{*}{$2,026,787$} & Jalandhar & 81.31 & 99.88 \\
\hline & & & Ludhiana & 0.07 & 0.06 \\
\hline & & & Nawanshahr & 18.62 & 71.06 \\
\hline
\end{tabular}


Table 6c. Boundary Changes in "Other" Districts: 1991-2001

\begin{tabular}{|c|c|c|c|c|c|}
\hline Census 1991 State/UT & 1991 District & $\begin{array}{c}\text { Population in } \\
1991\end{array}$ & 2001 District & $\begin{array}{c}\text { Share of } \\
2001 \\
\text { District in } \\
1991 \\
\text { District }\end{array}$ & $\begin{array}{c}\text { Share of } \\
1991 \\
\text { District in } \\
2001 \\
\text { District }\end{array}$ \\
\hline [1] & [2] & [3] & {$[4]$} & [5] & {$[6]$} \\
\hline \multirow{12}{*}{ Punjab } & \multirow{3}{*}{ Ludhiana } & \multirow{3}{*}{$2,471,594$} & Fatehgarh Sahib & 2.82 & 15.34 \\
\hline & & & Ludhiana & 97.06 & 98.79 \\
\hline & & & Rupnagar & 0.12 & 0.32 \\
\hline & \multirow{4}{*}{ Patiala } & \multirow{4}{*}{$1,896,242$} & Fatehgarh Sahib & 19.17 & 79.89 \\
\hline & & & Ludhiana & 0.08 & 0.06 \\
\hline & & & Patiala & 80.58 & 99.96 \\
\hline & & & Rupnagar & 0.18 & 0.38 \\
\hline & \multirow{3}{*}{ Rupnagar } & \multirow{3}{*}{915,603} & Fatehgarh Sahib & 2.37 & 4.77 \\
\hline & & & Patiala & 0.07 & 0.04 \\
\hline & & & Rupnagar & 97.56 & 99.30 \\
\hline & \multirow{2}{*}{ Sangrur } & \multirow{2}{*}{$1,710,120$} & Ludhiana & 1.44 & 1.02 \\
\hline & & & Sangrur & 98.56 & 100.00 \\
\hline \multirow{15}{*}{ Rajasthan } & Alwar & $2,296,580$ & Alwar & 100.00 & 100.00 \\
\hline & Bundi & 770,248 & Bundi & 100.00 & 100.00 \\
\hline & Chittorgarh & $1,484,190$ & Chittaurgarh & 100.00 & 100.00 \\
\hline & \multirow{2}{*}{ Jaipur } & \multirow{2}{*}{$4,722,551$} & Dausa & 17.67 & 83.93 \\
\hline & & & Jaipur & 82.33 & 100.00 \\
\hline & Jaisalmer & 344,517 & Jaisalmer & 100.00 & 100.00 \\
\hline & Jhalawar & 956,971 & Jhalawar & 100.00 & 100.00 \\
\hline & Jodhpur & $2,153,483$ & Jodhpur & 100.00 & 100.00 \\
\hline & \multirow{2}{*}{ Kota } & \multirow{2}{*}{$2,030,831$} & Baran & 39.90 & 100.00 \\
\hline & & & Kota & 60.10 & 100.00 \\
\hline & \multirow{3}{*}{ Sawai Madhopur } & \multirow{3}{*}{$1,963,246$} & Dausa & 8.14 & 16.07 \\
\hline & & & Karauli & 47.25 & 100.00 \\
\hline & & & Sawai Madhopur & 44.61 & 100.00 \\
\hline & \multirow{2}{*}{ Udaipur } & \multirow{2}{*}{$2,889,301$} & Rajsamand & 28.47 & 100.00 \\
\hline & & & Udaipur & 71.53 & 100.00 \\
\hline \multirow{2}{*}{ Sikkim } & South District & 98,604 & South & 100.00 & 100.00 \\
\hline & West District & 98,161 & West & 100.00 & 100.00 \\
\hline \multirow{15}{*}{ Tamil Nadu } & \multirow{2}{*}{ Chengalpattu-MGR } & 4653593 & Kancheepuram & 51.90 & 100.00 \\
\hline & & $4,653,593$ & Thiruvallur & 48.10 & 100.00 \\
\hline & Kamarajar & $1,565,037$ & Virudhunagar & 100.00 & 100.00 \\
\hline & Madras & $3,841,396$ & Chennai & 100.00 & 100.00 \\
\hline & Pasumpon M. Thevar & $1,078,190$ & Sivaganga & 100.00 & 97.74 \\
\hline & Puddukkottai & $1,327,148$ & Pudukkottai & 100.00 & 100.00 \\
\hline & lRamanathanuram & 1144040 & Ramanathapuram & 97.82 & 100.00 \\
\hline & namanatrapuram & $1,144,040$ & Sivaganga & 2.18 & 2.26 \\
\hline & & & Nagapattinam & 30.40 & 100.00 \\
\hline & Thanjavur & $4,531,457$ & Thanjavur & 45.32 & 100.00 \\
\hline & & & Thiruvarur & 24.28 & 100.00 \\
\hline & & & Ariyalur & 15.38 & 100.00 \\
\hline & Tiruchiranalli & 4138048 & Karur & 20.64 & 100.00 \\
\hline & III uctili apaili & $4,138,048$ & Perambalur & 10.90 & 100.00 \\
\hline & & & Tiruchirappalli & 53.08 & 100.00 \\
\hline & North Trinura & & Dhalai & 33.01 & 82.96 \\
\hline & Ivorth Iripura & 091,300 & North Tripura & 66.99 & 100.00 \\
\hline Tripura & South Trinura & 766014 & Dhalai & 6.17 & 17.04 \\
\hline & soutn Inpura & 106,014 & South Tripura & 93.83 & 100.00 \\
\hline & West Tripura & $1,293,861$ & West Tripura & 100.00 & 100.00 \\
\hline
\end{tabular}


Table 6c. Boundary Changes in "Other" Districts: 1991-2001

\begin{tabular}{|c|c|c|c|c|c|}
\hline Census 1991 State/UT & 1991 District & $\begin{array}{c}\text { Population in } \\
1991\end{array}$ & 2001 District & $\begin{array}{c}\text { Share of } \\
2001 \\
\text { District in } \\
1991 \\
\text { District }\end{array}$ & $\begin{array}{c}\text { Share of } \\
1991 \\
\text { District in } \\
2001 \\
\text { District }\end{array}$ \\
\hline [1] & [2] & [3] & [4] & [5] & {$[6]$} \\
\hline \multirow{54}{*}{ Uttar Pradesh } & Agra & $2,751,021$ & Agra & 100.00 & 100.00 \\
\hline & \multirow{2}{*}{ Aligarh } & \multirow{2}{*}{$3,295,982$} & Aligarh & 74.32 & 100.00 \\
\hline & & & Hathras & 25.68 & 75.11 \\
\hline & \multirow{2}{*}{ Almora } & \multirow{2}{*}{836,617} & Almora & 72.70 & 100.00 \\
\hline & & & Bageshwar & 27.30 & 100.00 \\
\hline & \multirow{2}{*}{ Azamgarh } & \multirow{2}{*}{$3,153,885$} & Ambedkar Nagar & 0.80 & 1.55 \\
\hline & & & Azamgarh & 99.20 & 100.00 \\
\hline & Ballia & $2,262,273$ & Ballia & 100.00 & 100.00 \\
\hline & \multirow{2}{*}{ Barabanki } & \multirow{2}{*}{$2,423,136$} & Barabanki & 87.19 & 100.00 \\
\hline & & & Faizabad & 12.81 & 18.42 \\
\hline & \multirow{2}{*}{ Basti } & \multirow{2}{*}{$2,738,522$} & Basti & 61.58 & 100.00 \\
\hline & & & Sant Kabir Nagar & 38.42 & 91.33 \\
\hline & Bijnor & $2,454,521$ & Bijnor & 100.00 & 100.00 \\
\hline & \multirow{2}{*}{ Bulandshahr } & \multirow{2}{*}{$2,849,859$} & Bulandshahar & 86.61 & 100.00 \\
\hline & & & Gautam Buddha Nagar & 13.39 & 44.89 \\
\hline & \multirow{2}{*}{ Chamoli } & \multirow{2}{*}{454,871} & Chamoli & 71.50 & 100.00 \\
\hline & & & Rudraprayag & 28.50 & 64.65 \\
\hline & \multirow{2}{*}{ Etawah } & \multirow{2}{*}{$2,124,655$} & Auraiya & 47.07 & 100.00 \\
\hline & & & Etawah & 52.93 & 99.49 \\
\hline & \multirow{2}{*}{ Faizabad } & 2078484 & Ambedkar Nagar & 53.86 & 98.45 \\
\hline & & $2,9 / 8,484$ & Faizabad & 46.14 & 81.58 \\
\hline & Fatehpur & $1,899,241$ & Fatehpur & 100.00 & 99.99 \\
\hline & Firozabad & $1,533,054$ & Firozabad & 100.00 & 100.00 \\
\hline & & & Garhwal & 98.29 & 100.00 \\
\hline & Garhwal & 682,535 & Nainital & 0.10 & 0.12 \\
\hline & & & Rudraprayag & 1.61 & 5.48 \\
\hline & Ghaziahad & & Gautam Buddha Nagar & 17.32 & 55.11 \\
\hline & Gnazıabad & $2,103,933$ & Ghaziabad & 82.68 & 100.00 \\
\hline & Hardwar & $1,124,488$ & Hardwar & 100.00 & 100.00 \\
\hline & IKannur Rehat & 2138317 & Kanpur Dehat & 60.95 & 100.00 \\
\hline & Kanpur Denat & $2, \mid 38,31 /$ & Kanpur Nagar & 39.05 & 25.67 \\
\hline & Kanpur Nagar & $2,418,487$ & Kanpur Nagar & 100.00 & 74.33 \\
\hline & Mainnuri & 1316746 & Etawah & 0.43 & 0.51 \\
\hline & |viainpuri & $1,316, / 46$ & Mainpuri & 99.57 & 100.00 \\
\hline & & 1931186 & Hathras & 14.53 & 24.89 \\
\hline & Iviatnura & $1,931,186$ & Mathura & 85.47 & 100.00 \\
\hline & Mau & $1,445,782$ & Mau & 100.00 & 100.00 \\
\hline & Meerut & 3447912 & Baghpat & 29.88 & 100.00 \\
\hline & Ivieerut & $3,447,912$ & Meerut & 70.12 & 100.00 \\
\hline & Muzaffarnagar & $2,842,543$ & Muzaffarnagar & 100.00 & 100.00 \\
\hline & & & Champawat & 2.67 & 21.56 \\
\hline & Nainital & $1,540,174$ & Nainital & 37.28 & 99.88 \\
\hline & & & Udham Singh Nagar & 60.05 & 100.00 \\
\hline & Pithoragarh & 566408 & Champawat & 26.44 & 78.44 \\
\hline & |ritrioragam & 506,408 & Pithoragarh & 73.56 & 100.00 \\
\hline & & & Fatehpur & 0.01 & 0.01 \\
\hline & Rae Bareli & $2,322,810$ & Rae Bareli & 99.45 & 100.00 \\
\hline & & & Sultanpur & 0.55 & 0.50 \\
\hline & Saharanpur & $2,309,029$ & Saharanpur & 100.00 & 100.00 \\
\hline & Siddharth Nagar & 1707885 & Sant Kabir Nagar & 5.85 & 8.67 \\
\hline & siaunartn Ivagar & & Siddharthnagar & 94.15 & 100.00 \\
\hline & Sultanpur & $2,558,970$ & Sultanpur & 100.00 & 99.50 \\
\hline & Tehri Garhwal & 580153 & Rudraprayag & 10.32 & 29.87 \\
\hline & renr Gamwal & 500,150 & Tehri Garhwal & 89.68 & 100.00 \\
\hline
\end{tabular}


Table 6c. Boundary Changes in "Other" Districts: 1991-2001

\begin{tabular}{|c|c|c|c|c|c|}
\hline Census 1991 State/UT & 1991 District & $\begin{array}{c}\text { Population in } \\
1991\end{array}$ & 2001 District & $\begin{array}{c}\text { Share of } \\
2001 \\
\text { District in } \\
1991 \\
\text { District }\end{array}$ & $\begin{array}{c}\text { Share of } \\
1991 \\
\text { District in } \\
2001 \\
\text { District }\end{array}$ \\
\hline$[1]$ & [2] & {$[3]$} & {$[4]$} & [5] & {$[6]$} \\
\hline \multirow{5}{*}{ West Bengal } & Calcutta & $4,399,819$ & Kolkata & 100.00 & 100.00 \\
\hline & Murshidabad & $4,740,149$ & Murshidabad & 100.00 & 100.00 \\
\hline & Nadia & $3,852,097$ & Nadia & 100.00 & 100.00 \\
\hline & North 24 Paraganas & $7,281,881$ & North Twenty Four Parganas & 100.00 & 100.00 \\
\hline & South 24 Paraganas & $5,715,030$ & South Twenty Four Parganas & 100.00 & 100.00 \\
\hline
\end{tabular}

* Census operations were not held in Jammu \& Kashmir in 1991 due to political unrest. The population figures reported for this state in 1991 are interpolated numbers reported in Census 2001 documents.

a an uninhabited portion of one village, with an area of $0.07 \mathrm{sq}$. km., was transferred from Alappuzha district to Pathanamthitta district in 1996.

${ }^{b}$ uninhabited portions of two villages, with a total area of $0.33 \mathrm{sq} . \mathrm{km}$., were transferred from Ernakulam district to Idukki district in 1997.

${ }^{c}$ an uninhabited portion of one village, with an area of $0.01 \mathrm{sq} . \mathrm{km}$., was transferred from Kollam district to Pathanamthitta district in 1992.

${ }^{d}$ an uninhabited portion of one village, with an area of 5.03 sq. km., was transferred from Pathanamthitta district to Kottayam district in 1995. 
Table 7. The Number of Consistent Regions between each Census Year and 2001

For each state, this table counts the number of regions with unchanged boundaries that can be used for panels between 2001 and each census year 1971, 1981 and 1991. This includes districts that are unchanged or partitioned, as well as the composite regions listed in Table 8.

\begin{tabular}{|c|c|c|c|}
\hline \multirow[t]{2}{*}{ Census 2001 State/UT } & \multicolumn{3}{|c|}{ Number of Regions } \\
\hline & 1971 & 1981 & 1991 \\
\hline [1] & [2] & [3] & [4] \\
\hline Andaman \& Nicobar Islands & 1 & 2 & 2 \\
\hline Andhra Pradesh ${ }^{\mathrm{a}}$ & 18 & 21 & 22 \\
\hline Arunachal Pradesh & 4 & 8 & 11 \\
\hline Assam & 6 & 6 & 22 \\
\hline Bihar & 10 & 23 & 28 \\
\hline Chandigarh & 1 & 1 & 1 \\
\hline Chhattisgarh & 5 & 6 & 6 \\
\hline Dadra \& Nagar Haveli & 1 & 1 & 1 \\
\hline Daman \& Diu & 2 & 2 & 2 \\
\hline Delhi & 1 & 1 & 1 \\
\hline Goa & 1 & 1 & 2 \\
\hline Gujarat & 12 & 12 & 12 \\
\hline Haryanab & 1 & 3 & 6 \\
\hline Himachal Pradesh & 7 & 11 & 12 \\
\hline Jammu \& Kashmir & 7 & 14 & 14 \\
\hline Jharkhand & 5 & 6 & 12 \\
\hline Karnataka & 16 & 16 & 18 \\
\hline Kerala & 4 & 8 & 13 \\
\hline Lakshadweep & 1 & 1 & 1 \\
\hline Madhya Pradesh & 35 & 37 & 37 \\
\hline Maharashtra $^{\mathrm{a}}$ & 20 & 20 & 30 \\
\hline Manipur & 2 & 4 & 8 \\
\hline Meghalaya & 2 & 4 & 4 \\
\hline Mizoram & 1 & 3 & 3 \\
\hline Nagaland & 2 & 4 & 7 \\
\hline Orissa & 13 & 13 & 13 \\
\hline Pondicherry & 4 & 4 & 4 \\
\hline Punjab & 2 & 3 & 3 \\
\hline Rajasthan & 20 & 23 & 26 \\
\hline Sikkim & 3 & 4 & 4 \\
\hline Tamil Nadu & 12 & 16 & 20 \\
\hline Tripura & 1 & 2 & 2 \\
\hline Uttar Pradesh ${ }^{b, c}$ & 35 & 37 & 45 \\
\hline Uttaranchal $^{c}$ & 4 & 5 & 5 \\
\hline West Bengal & 14 & 15 & 17 \\
\hline India & 267 & 334 & 414 \\
\hline
\end{tabular}

a In 1971 and 1981, one composite region cuts across the state boundaries of Andhra Pradesh and Maharashtra.

${ }^{\mathrm{b}}$ In 1971 and 1981, one composite region cuts across the state boundaries of Haryana and Uttar Pradesh.

${ }^{\mathrm{C}}$ In 1971 and 1981, one composite region cuts across the state boundaries of Uttar Pradesh and Uttaranchal.

Notes:

1. When a region cuts across state boundaries, it is included in the count of regions for each of those states. However, the totals for India do not double-count such regions. 


\begin{tabular}{|c|c|c|c|c|c|}
\hline \multicolumn{6}{|c|}{ Table 8. Composite Regions } \\
\hline 1971 State/UT & 1971 Region & 1981 Region & 1991 Region & 2001 District & 2001 State/UT \\
\hline$[1]$ & {$[2]$} & {$[3]$} & {$[4]$} & [5] & \begin{tabular}{|r}
{$[6]$} \\
\end{tabular} \\
\hline \multirow{9}{*}{ Andhra Pradesh } & Adilabad, Chandrapur ${ }^{\mathrm{a}}$ & Adilabad, Chandrapur ${ }^{a}$ & Adilabad & Adilabad & \multirow{9}{*}{ Andhra Pradesh } \\
\hline & Anantapur, Cuddapah & $\begin{array}{l}\text { Anantapuram, } \\
\text { Cuddapah }\end{array}$ & Anantapur, Cuddapah & \begin{tabular}{|l} 
Anantapur \\
Cuddapah
\end{tabular} & \\
\hline & \multirow{2}{*}{ Chittoor, Nellore } & Chittoor & Chittoor & Chittoor & \\
\hline & & Nellore & Nellore & Nellore & \\
\hline & \multirow{2}{*}{ Hyderabad } & Hyderabad, & Hyderabad & Hyderabad & \\
\hline & & Rangareddy & Rangareddi & Rangareddi & \\
\hline & \multirow{3}{*}{$\begin{array}{l}\text { Srikakulam, } \\
\text { Visakhapatnam }\end{array}$} & Srikakulam & Srikakulam & Srikakulam & \\
\hline & & Vishakhapatanam & Visakhapatanam & Visakhapatnam & \\
\hline & & Vizianagaram & Vizianagaram & Vizianagaram & \\
\hline \multirow{6}{*}{ Arunachal Pradesh } & \multirow{6}{*}{ Siang, Subansiri } & \multirow{3}{*}{ East Siang, West Siang } & East Siang & East Siang & \multirow{6}{*}{ Arunachal Pradesh } \\
\hline & & & East slang & Upper Siang & \\
\hline & & & West Siang & West Siang & \\
\hline & & \multirow{2}{*}{ Lower Subansiri } & \multirow{2}{*}{ Lower Subansiri } & Lower Subansiri & \\
\hline & & & & Papum Pare & \\
\hline & & Upper Subansiri & Upper Subansiri & Upper Subansiri & \\
\hline \multirow{14}{*}{ Assam } & \multirow{5}{*}{ Darrang, Sibsagar } & & Darrang & Darrang & \\
\hline & & & Golaghat & Golaghat & \\
\hline & & Darrang, Sibsagar & Jorhat & Jorhat & \\
\hline & & & Sibsagar & Sibsagar & \\
\hline & & & Sonitpur & Sonitpur & \\
\hline & & & Barpeta & Barpeta & \\
\hline & & & Bongaigaon & Bongaigaon & \\
\hline & & & & Dhubri & Assam \\
\hline & & & Dhubri, Kokrajhar & Kokrajhar & \\
\hline & Goalpara, Kamrup, & Goalpara, Kamrup, & Goalpara & Goalpara & \\
\hline & & & Kamrup & Kamrup & \\
\hline & & & Marigaon & Marigaon & \\
\hline & & & Nagaon & Nagaon & \\
\hline & & & Nalbari & Nalbari & \\
\hline & & Begusarai & Begusarai & Begusarai & \\
\hline & & & Khagaria & Khagaria & \\
\hline & & & & Jamui & \\
\hline & Monahvr Patna & Munger Nalanda & & Lakhisarai & Bihar \\
\hline & |Viongnyr, Patna & |viunger, Nalanda & Munger, Nalanda & Munger & Binar \\
\hline & & & & Nalanda & \\
\hline & & & & Sheikhpura & \\
\hline Bihar & & Patna & Patna & Patna & \\
\hline & & & & Bokaro & \\
\hline & & Dhanbad, Giridih & Dhanbad, Giridih & Dhanbad & \\
\hline & Dhanbad Hazaribagh & & & Giridih & Tharkhand \\
\hline & & & & Chatra & Jnarknana \\
\hline & & Hazaribagh & Hazaribag & Hazaribagh & \\
\hline & & & & Kodarma & \\
\hline & & & & Ahmadabad & \\
\hline & & & & Anand & \\
\hline & Ahmadabad, Banas & Ahmadabad, Banas & Ahmadabad, Banas & Banas Kantha & \\
\hline & Kantha, Gandhinagar, & Kantha, Gandhinagar, & Kantha, Gandhinagar, & Gandhinagar & \\
\hline & Kheda (Kaira), Mehsana & Kheda, Mahesana & Kheda, Mahasana & Kheda & \\
\hline & & & & Mahesana & \\
\hline Guiarat & & & & Patan & Guiarat \\
\hline Gujarat & & & & Amreli & Gujarat \\
\hline & Amreli, Bhavnagar, & Amreli, Bhavnagar, & Amreli, Bhavnagar, & Bhavnagar & \\
\hline & Junagadh & Junagadh & Junagadh & Junagadh & \\
\hline & & & & Porbandar & \\
\hline & & & & Bharuch & \\
\hline & Vadodara (Baroda) & Bharuch, Vadodara & Bharuch, Vadodara & Narmada & \\
\hline & & & & Vadodara & \\
\hline
\end{tabular}




\begin{tabular}{|c|c|c|c|c|c|}
\hline \multicolumn{6}{|c|}{ Table 8. Composite Regions } \\
\hline 1971 State/UT & 1971 Region & 1981 Region & 1991 Region & 2001 District & 2001 State/UT \\
\hline$[1]$ & {$[2]$} & {$[3]$} & {$[4]$} & {$[5]$} & {$[6]$} \\
\hline \multirow{18}{*}{ Haryana } & \multirow{18}{*}{$\begin{array}{l}\text { Ambala, Bulandshahr, } \\
\text { Gurgaon, Hisar, Jind, } \\
\text { Karnal, Mahendragarh, } \\
\text { Meerut, Rohtak }{ }^{\mathrm{b}}\end{array}$} & \multirow{15}{*}{$\begin{array}{l}\text { Ambala, Bhiwani, Hisar } \\
\text { Jind, Karnal, } \\
\text { Kurukshetra, } \\
\text { Mahendragarh, Rohtak, } \\
\text { Sonipat }\end{array}$} & Ambala & \begin{tabular}{|l|} 
Ambala \\
Panchkula
\end{tabular} & \multirow{18}{*}{ Haryana } \\
\hline & & & \multirow{13}{*}{$\begin{array}{l}\text { Bhiwani, Hisar, Jind, } \\
\text { Kaithal, Karnal, } \\
\text { Kurukshetra, Panipat, } \\
\text { Rewari, Rohtak, Sonipat, } \\
\text { Yamunanagar }\end{array}$} & Bhiwani & \\
\hline & & & & Fatehabad & \\
\hline & & & & Hisar & \\
\hline & & & & Jhaijar & \\
\hline & & & & Jind & \\
\hline & & & & Kaithal & \\
\hline & & & & Karnal & \\
\hline & & & & Kurukshetra & \\
\hline & & & & Panipat & \\
\hline & & & & Rewari & \\
\hline & & & & Rohtak & \\
\hline & & & & Sonipat & \\
\hline & & & & Yamunanagar & \\
\hline & & & Mahendragarh & Mahendragarh & \\
\hline & & \multirow{2}{*}{$\begin{array}{l}\text { Bulandshahr, } \\
\text { Faridabad, Ghaziabad, } \\
\text { Gurgaon }^{\text {b }}\end{array}$} & Faridabad & Faridabad & \\
\hline & & & Gurgaon & Gurgaon & \\
\hline & & Sirsa & Sirsa & Sirsa & \\
\hline \multirow{5}{*}{ Himachal Pradesh } & \multirow{2}{*}{ Chamba, Lahul \& Spiti } & Chamba & Chamba & Chamba & \multirow{5}{*}{ Himachal Pradesh } \\
\hline & & Lahul And Spiti & Lahul And Spiti & Lahul \& Spiti & \\
\hline & \multirow{3}{*}{ Mahasu, Simla, Sirmaur } & Shimla & Shimla & Shimla & \\
\hline & & \multirow{2}{*}{ Sirmaur, Solan } & Sirmaur & Sirmaur & \\
\hline & & & Solan & Solan & \\
\hline \multirow{7}{*}{ Jammu \& Kashmir } & \multirow{4}{*}{ Baramula, Srinagar } & Badgam & Badgam & Badgam & \multirow{7}{*}{ Jammu \& Kashmi } \\
\hline & & $\begin{array}{l}\text { Baramula (Kashmir } \\
\text { North) }\end{array}$ & Baramula & Baramula & \\
\hline & & Kupwara & Kupwara & Kupwara & \\
\hline & & Srinagar & Srinagar & Srinagar & \\
\hline & \multirow{3}{*}{$\begin{array}{l}\text { Jammu, Kathua, } \\
\text { Udhampur }\end{array}$} & Jammu & Jammu & Jammu & \\
\hline & & Kathua & Kathua & Kathua & \\
\hline & & Udhampur & Udhampur & Udhampur & \\
\hline \multirow{13}{*}{ Kerala } & & & Alappuzha & Alappuzha & \\
\hline & & & Frnakulam Idukki & Ernakulam & \\
\hline & & Alleppey, Ernakulam, & & Idukki & \\
\hline & Alleppey, Ernakulam, & Idukki, Quilon, Trichur & Kollam & Kollam & \\
\hline & & & Pathanamthitta & Pathanamthitta & \\
\hline & & & Thrissur & Thrissur & \\
\hline & & Kottayam & Kottayam & Kottayam & Kerala \\
\hline & & lCannanore & Kannur & Kannur & \\
\hline & I Cannonera Kazhiknade & Jannanore & Kasaragod & Kasaragod & \\
\hline & |Cannanore, Kozhikode & Kozhikode & Kozhikode & Kozhikode & \\
\hline & & Wayanad & Wayanad & Wayanad & \\
\hline & Mallanuram Palahat & Malappuram & Malappuram & Malappuram & \\
\hline & |Mallapuram, Palghat & Palghat & Palakkad & Palakkad & \\
\hline & & & & Bilaspur & \\
\hline & & & & Janjgir - Champa & \\
\hline & & Bilaspur, Raj Nandgaon & Bilaspur, Rajnandgaon & Kawardha & \\
\hline & |Bilaspur, Durg & & & Korba & Chhattisgarh \\
\hline & & & & Rajnandgaon & \\
\hline Madhva Pradesh & & Durg & Durg & Durg & \\
\hline & Datia Gualinr & Datia Gualing & Datia Gualior & Datia & \\
\hline & Lalla, Gwallor & jDalla, Gwallor & |Lalla, Gwallor & Gwalior & \\
\hline & & Bhopal & Bhopal & Bhopal & Madbva Pradech \\
\hline & LHechanarhed Sohore & LHechengahed & Hechangahod & Harda & IMadhya Pradesn \\
\hline & Hoshangabad, Sehore & Hoshangabad & Hoshangabad & Hoshangabad & \\
\hline & & Sehore & Sehore & Sehore & \\
\hline
\end{tabular}




\begin{tabular}{|c|c|c|c|c|c|}
\hline \multicolumn{6}{|c|}{ Table 8. Composite Regions } \\
\hline 1971 State/UT & 1971 Region & 1981 Region & 1991 Region & 2001 District & 2001 State/UT \\
\hline$[1]$ & {$[2]$} & {$[3]$} & {$[4]$} & {$[5]$} & {$[6]$} \\
\hline \multirow{15}{*}{ Maharashtra } & \multirow{2}{*}{ Adilabad, Chandrapur ${ }^{a}$} & \multirow{2}{*}{ Adilabad, Chandrapur } & Chandrapur & Chandrapur & \multirow{15}{*}{ Maharashtra } \\
\hline & & & Gadchiroli & Gadchiroli & \\
\hline & \multirow{5}{*}{$\begin{array}{l}\text { Ahmednagar, } \\
\text { Aurangabad, Parbhani }\end{array}$} & \multirow{5}{*}{$\begin{array}{l}\text { Ahmadnagar, } \\
\text { Aurangabad, Parbhani }\end{array}$} & Ahmednagar & Ahmadnagar & \\
\hline & & & Aurangabad & Aurangabad & \\
\hline & & & Jalna & Jalna & \\
\hline & & & \multirow{2}{*}{ Parbhani } & Hingoli & \\
\hline & & & & Parbhani & \\
\hline & \multirow{5}{*}{$\begin{array}{l}\text { Bhir, Osmanabad, Sangli, } \\
\text { Sholapur }\end{array}$} & \multirow{5}{*}{$\begin{array}{l}\text { Bid, Osmanabad, } \\
\text { Sangli, Solapur }\end{array}$} & Bid & Bid & \\
\hline & & & Latur & Latur & \\
\hline & & & Osmanabad & Osmanabad & \\
\hline & & & Sangli & Sangli & \\
\hline & & & Solapur & Solapur & \\
\hline & \multirow{3}{*}{ Kolhapur, Ratnagiri } & \multirow{3}{*}{ Kolhapur, Ratnagiri } & Kolhapur & Kolhapur & \\
\hline & & & Ratnagiri & Ratnagiri & \\
\hline & & & Sindhudurg & Sindhudurg & \\
\hline \multirow{8}{*}{ Manipur } & \multirow{8}{*}{$\begin{array}{l}\text { Manipur Central, Manipur } \\
\text { North, Manipur South, } \\
\text { Manipur West }\end{array}$} & \multirow{6}{*}{$\begin{array}{l}\text { Manipur Central, } \\
\text { Manipur North, Manipur } \\
\text { South }\end{array}$} & Bishnupur & Bishnupur & \multirow{8}{*}{ Manipur } \\
\hline & & & Churachandpur & Churachandpur & \\
\hline & & & Imnhal & Imphal East & \\
\hline & & & IImpnal & Imphal West & \\
\hline & & & Senapati & Senapati & \\
\hline & & & Thoubal & Thoubal & \\
\hline & & Manipur West & Tamenglong & Tamenglong & \\
\hline & & Tengnoupal & Chandel & Chandel & \\
\hline & & East Khasi Hills. West & East Khasi Hills. West & East Khasi Hills & \\
\hline Meghalaya & United Khasi \& Jaintia & Khasi Hills & $\begin{array}{l}\text { Last n } \\
\text { Khasi Hills }\end{array}$ & Ri Bhoi & Meahalaya \\
\hline & & & & West Khasi Hills & IVleginalaya \\
\hline & & Jaintia Hills & Jaintia Hills & Jaintia Hills & \\
\hline & Reloaum North Kanara & Relaaum Uttar Kannad & Belgaum & Belgaum & \\
\hline & |Beigaum, Nortn Kanara & & Uttar Kannad & Uttara Kannada & \\
\hline Mysore & & & & Bellary & Karnataka \\
\hline |Viysore & Bellary, Chitradurga, & Bellary, Chitradurga, & Bellary, Chitradurga, & Chitradurga & Karnataka \\
\hline & Shimoga & Shimoga & Shimoga & Davanagere & \\
\hline & & & & Shimoga & \\
\hline & & & Kohima & Dimapur & \\
\hline & & Kohima, Zunheboto & Konima & Kohima & \\
\hline & Kohima Mokokchung & & Zunheboto & Zunheboto & \\
\hline Nagaland & INonima, IVIOKOKCnung & Mokokrhung Wokha & Mokokchung & Mokokchung & Nagaland \\
\hline INagalaria & & 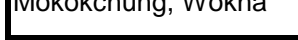 & Wokha & Wokha & INagalantu \\
\hline & & Phek & Phek & Phek & \\
\hline & Tиencang & Mon Tuncang & Mon & Mon & \\
\hline & puensang & fivion, i luensang & Tuensang & Tuensang & \\
\hline & & & & Amritsar & \\
\hline & & & & Faridkot & \\
\hline & & & & Fatehgarh Sahib & \\
\hline & & & & Firozpur & \\
\hline & & & & Gurdaspur & \\
\hline & Amritcor Photinda & Amritsar, rarıdkot, & Amritsar, raridkot, & Hoshiarpur & \\
\hline & $\begin{array}{l}\text { Amritsar, Bnatinaa, } \\
\text { Firozpur, Gurdaspur, }\end{array}$ & Hoshiarpur, Jalandhar, & Hoshiarpur, Jalandhar, & Jalandhar & \\
\hline Punjab & Hoshiarpur, Jullundur, & Ludhiana, Patiala, & Ludhiana. Patiala. & Ludhiana & Puniab \\
\hline & Ludhiana, Patiala, Ropar, & Rupnagar, Sangrur & Rupnagar, Sangrur & Moga & \\
\hline & Sangrur & & & Muktsar & \\
\hline & & & & Nawanshahr & \\
\hline & & & & Patiala & \\
\hline & & & & Rupnagar & \\
\hline & & & & Sangrur & \\
\hline & & Rathinda & Rathinda & Bathinda & \\
\hline & & |Bathinda & |Batninda & Mansa & \\
\hline
\end{tabular}




\begin{tabular}{|c|c|c|c|c|c|}
\hline \multicolumn{6}{|c|}{ Table 8. Composite Regions } \\
\hline 1971 State/UT & 1971 Region & 1981 Region & 1991 Region & 2001 District & 2001 State/UT \\
\hline $\begin{array}{r}{[1]} \\
\end{array}$ & {$[2]$} & {$[3]$} & {$[4]$} & {$[5]$} & \begin{tabular}{|c|}
{$[6]$} \\
\end{tabular} \\
\hline \multirow{14}{*}{ Rajasthan } & \multirow{5}{*}{$\begin{array}{l}\text { Alwar, Jaipur, Sawai } \\
\text { Madhopur }\end{array}$} & \multirow{5}{*}{$\begin{array}{l}\text { Alwar, Jaipur, Sawai } \\
\text { Madhopur }\end{array}$} & Alwar & Alwar & \multirow{14}{*}{ Rajasthan } \\
\hline & & & \multirow{4}{*}{ Jaipur, Sawai Madhopur } & Dausa & \\
\hline & & & & Jaipur & \\
\hline & & & & Karauli & \\
\hline & & & & Sawai Madhopur & \\
\hline & \multirow{4}{*}{ Bundi, Jhalawar, Kota } & \multirow{3}{*}{ Bundi, Kota } & Bundi & Bundi & \\
\hline & & & \multirow{2}{*}{ Kota } & Baran & \\
\hline & & & & Kota & \\
\hline & & Jhalawar & Jhalawar & Jhalawar & \\
\hline & \multirow{3}{*}{ Chittaurgarh, Udaipur } & Chittaurgarh & Chittorgarh & Chittaurgarh & \\
\hline & & \multirow{2}{*}{ Udaipur } & \multirow{2}{*}{ Udaipur } & Rajsamand & \\
\hline & & & & Udaipur & \\
\hline & \multirow{2}{*}{ Jaisalmer, Jodhpur } & Jaisalmer & Jaisalmer & Jaisalmer & \\
\hline & & Jodhpur & Jodhpur & Jodhpur & \\
\hline \multirow{2}{*}{ Sikkim } & \multirow{2}{*}{$\begin{array}{l}\text { South District, West } \\
\text { District }\end{array}$} & South District & South District & South & \multirow{2}{*}{ Sikkim } \\
\hline & & West District & West District & West & \\
\hline \multirow{14}{*}{ Tamil Nadu } & & Chengalpattu & Chenaalpattu-MGR & Kancheepuram & \\
\hline & Chingleput, Madras & & & Thiruvallur & \\
\hline & & Madras & Madras & Chennai & \\
\hline & & & Kamarajar & Virudhunagar & \\
\hline & Ramanathapuram & Ramanathapuram & Pasumpon M. Thevar, & Ramanathapuram & \\
\hline & & & Ramanathapuram & Sivaganga & \\
\hline & & Pudukkottai & Puddukkottai & Pudukkottai & Tamil Nadu \\
\hline & & & & Nagapattinam & I amin ivauu \\
\hline & & Thanjavur & Thanjavur & Thanjavur & \\
\hline & IThaniavur Tiruchirapalli & & & Thiruvarur & \\
\hline & & & & Ariyalur & \\
\hline & & Tiruchchirapoalli & Tiruchirapalli & Karur & \\
\hline & & & & Perambalur & \\
\hline & & & & Tiruchirappalli & \\
\hline & & & & Dhalai & \\
\hline Tripura & North Tripura, South & North Tripura, South & $\begin{array}{l}\text { North I ripura, South } \\
\text { Tripura }\end{array}$ & North Tripura & Trinura \\
\hline 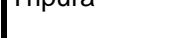 & Tripura, West Tripura & Tripura & & South Tripura & Iripura \\
\hline & & & West Tripura & West Tripura & \\
\hline & & & Agra & Agra & \\
\hline & & & & Auraiya & \\
\hline & Agra, Etawah, Mainpuri & Agra, Etawah, Mainpuri & Etawah, Mainpuri & Etawah & \\
\hline & & & & Mainpuri & \\
\hline & & & Firozabad & Firozabad & \\
\hline & & & & Aligarh & \\
\hline & Aligarh, Mathura & Aligarh, Mathura & Aligarh, Mathura & Hathras & \\
\hline & & & & Mathura & \\
\hline & & & & Bulandshahar & \\
\hline & $\begin{array}{l}\text { Ambala, Bulandshahr, } \\
\text { Gurgaon, Hisar, Jind, }\end{array}$ & Faridabad, Ghaziabad, & Bulandshahr, Ghaziabad & Gautam Buddha & \\
\hline Uttar Pradesh & Karnal, Mahendragarh, & Gurgaon ${ }^{\mathrm{b}}$ & & Ghagar & Uttar Pradesh \\
\hline & Meerut, Rohtak ${ }^{\mathrm{b}}$ & & & Baghpat & \\
\hline & & |Meerut & |Mleerut & Meerut & \\
\hline & & & & Ambedkar Nagar & \\
\hline & & & Azamgarh, Barabanki, & Azamgarh & \\
\hline & Azamgarh, Ballia, Bara & Azamgarh, Ballia, Bara & Faizabad & Barabanki & \\
\hline & Banki, Faizabad & Banki, Faizabad & & Faizabad & \\
\hline & & & Ballia & Ballia & \\
\hline & & & Mau & Mau & \\
\hline & & & & Basti & \\
\hline & Basti & Basti & Basti, Siddharth Nagar & Sant Kabir Nagar & \\
\hline & & & & Siddharthnagar & \\
\hline
\end{tabular}




\begin{tabular}{|c|c|c|c|c|c|}
\hline \multicolumn{6}{|c|}{ Table 8. Composite Regions } \\
\hline 1971 State/UT & 1971 Region & 1981 Region & 1991 Region & 2001 District & 2001 State/UT \\
\hline$[1]$ & {$[2]$} & {$[3]$} & {$[4]$} & {$[5]$} & $\begin{array}{r}6] \\
\end{array}$ \\
\hline \multirow{19}{*}{ Uttar Pradesh } & \multirow{3}{*}{$\begin{array}{l}\text { Fatehpur, Rae Bareli, } \\
\text { Sultanpur }\end{array}$} & \multirow{3}{*}{$\begin{array}{l}\text { Fatehpur, Rae Bareli, } \\
\text { Sultanpur }\end{array}$} & \multirow{3}{*}{$\begin{array}{l}\text { Fatehpur, Rae Bareli, } \\
\text { Sultanpur }\end{array}$} & Fatehpur & \multirow{8}{*}{ Uttar Pradesh } \\
\hline & & & & Rae Bareli & \\
\hline & & & & Sultanpur & \\
\hline & \multirow{2}{*}{ Kanpur } & \multirow{2}{*}{ Kanpur } & \multirow{2}{*}{$\begin{array}{l}\text { Kanpur Dehat, Kanpur } \\
\text { Nagar }\end{array}$} & Kanpur Dehat & \\
\hline & & & & Kanpur Nagar & \\
\hline & \multirow{4}{*}{$\begin{array}{l}\text { Bijnor, Muzaffarnagar, } \\
\text { Saharanpur }\end{array}$} & \multirow{4}{*}{$\begin{array}{l}\text { Bijnor, Muzaffarnagar, } \\
\text { Saharanpur }{ }^{c}\end{array}$} & Bijnor & Bijnor & \\
\hline & & & Muzaffarnagar & Muzaffarnagar & \\
\hline & & & Saharanpur & Saharanpur & \\
\hline & & & Hardwar & Hardwar & \multirow{11}{*}{ Uttaranchal } \\
\hline & \multirow{10}{*}{$\begin{array}{l}\text { Almora, Chamoli, } \\
\text { Garhwal, Naini Tal, } \\
\text { Pithoragarh, Tehri } \\
\text { Garhwal }\end{array}$} & \multirow{2}{*}{ Almora } & \multirow{2}{*}{ Almora } & Almora & \\
\hline & & & & Bageshwar & \\
\hline & & \multirow{8}{*}{$\begin{array}{l}\text { Chamoli, Garhwal, Naini } \\
\text { Tal, Pithoragarh, Tehri } \\
\text { Garhwal }\end{array}$} & \multirow{8}{*}{$\begin{array}{l}\text { Chamoli, Garhwal, } \\
\text { Nainital, Pithoragarh, } \\
\text { Tehri Garhwal }\end{array}$} & Chamoli & \\
\hline & & & & Champawat & \\
\hline & & & & Garhwal & \\
\hline & & & & Nainital & \\
\hline & & & & Pithoragarh & \\
\hline & & & & Rudraprayag & \\
\hline & & & & Tehri Garhwal & \\
\hline & & & & Udham Singh & \\
\hline \multirow{6}{*}{ West Bengal } & \multirow{4}{*}{$\begin{array}{l}\text { Calcutta, Twenty Four } \\
\text { Parganas }\end{array}$} & \multirow{4}{*}{$\begin{array}{l}\text { Calcutta, Twenty Four } \\
\text { Parganas }\end{array}$} & Calcutta & Kolkata & \multirow{6}{*}{ West Bengal } \\
\hline & & & Nerth 24 Parga & North Twenty Four & \\
\hline & & & |Nortn 24 Parganas & Parganas & \\
\hline & & & South 24 Parganas & $\begin{array}{l}\text { South Twenty Four } \\
\text { Parganas }\end{array}$ & \\
\hline & \multirow{2}{*}{ Murshidabad, Nadia } & Murshidabad & Murshidabad & Murshidabad & \\
\hline & & Nadia & Nadia & Nadia & \\
\hline
\end{tabular}

a This region cuts across the state boundaries of Andhra Pradesh and Maharashtra.

${ }^{\mathrm{b}}$ This region cuts across the state boundaries of Haryana and Uttar Pradesh.

${ }^{c}$ This region cuts across the state boundaries of Uttar Pradesh and Uttaranchal. 